NASA Technical Memorandum 105826

$$
\begin{aligned}
& 1 N-08 \\
& 17619 \\
& p-30
\end{aligned}
$$

\title{
A Parameter Optimization Approach to Controller Partitioning for Integrated Flight/Propulsion Control Application
}

Phillip Schmidt

The University of Akron

Akron, Ohio

Sanjay Garg

Lewis Research Center

Cleveland, Ohio
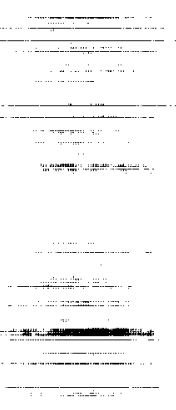

and

Brian Holowecky

The University of Akron

Akron, Ohio

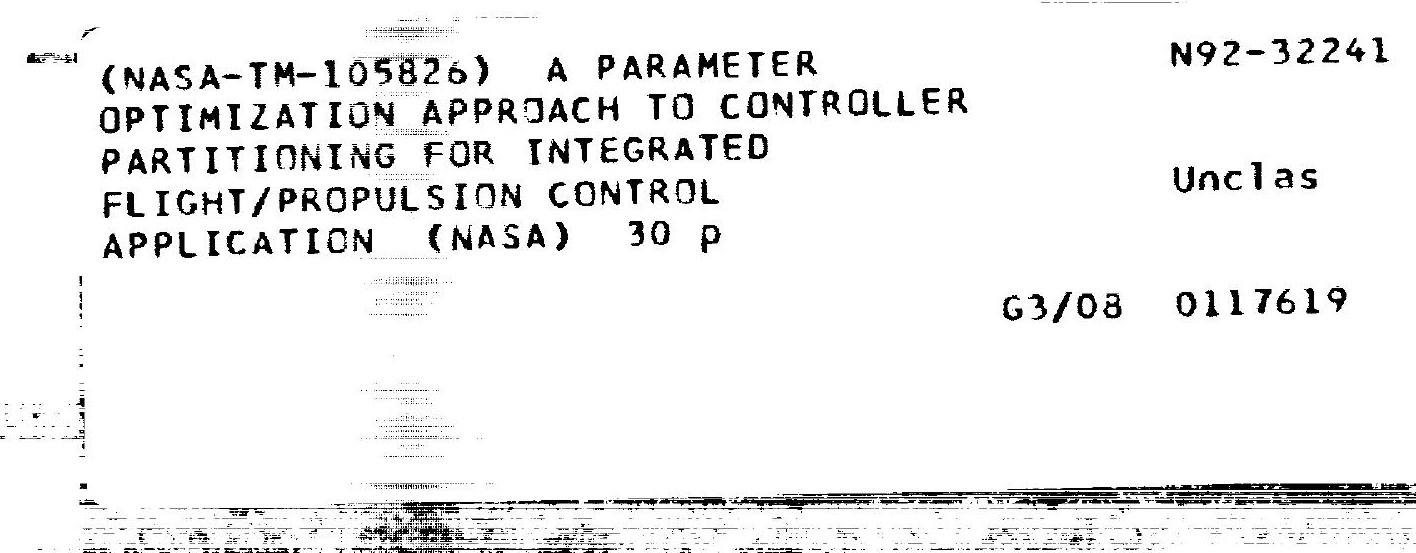




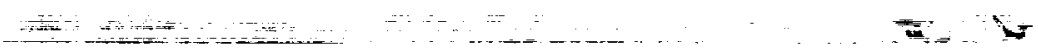




\title{
A Parameter Optimization Approach to Controller Partitioning for Integrated Flight/Propulsion Control Application
}

\author{
PhILLIP SCHMIDT ${ }^{1}$ \\ The University of Akron, Akron, Ohio \\ SANJAY GARG ${ }^{2}$ \\ NASA Lewis Research Center, Cleveland, Ohio \\ BRIAN HOLOWECKY ${ }^{3}$ \\ The University of Akron, Akron, Ohio
}

\begin{abstract}
A parameter optimization framework is presented to solve the problem of partitioning a centralized controller into a decentralized hierarchical structure suitable for integrated flight/propulsion control implementation. The controller partitioning problem is briefly discussed and a cost function to be minimized is formulated, such that the resulting "optimal" partitioned subsystem controllers will closely match the performance (including robustness) properties of the closed-loop system with the centralized controller while maintaining the desired controller partitioning structure. The cost function is written in terms of parameters in a state- space representation of the partitioned subcontrollers. Analytical expressions are obtained for the gradient of this cost function with respect to parameters and an optimization algorithm is developed using modern computer-aided control design and analysis software. The capabilities of the algorithm are demonstrated by application to partitioned integrated flight/propulsion control design for a modern fighter aircraft in the short approach to landing task. The partitioning optimization is shown to lead to reduced-order subcontrollers that match the closed-loop command tracking and decoupling performance achieved by a high-order centralized controller.
\end{abstract}

${ }^{1}$ Professor of Mathematical Sciences. The research of the first and third authors was supported by NASA grant NAG 3-1146

${ }^{2}$ Controls Engineer, Advanced Control Technology Branch

${ }^{3}$ Graduate Student, Electrical Engineering 


\section{INTRODUCTION}

Large interconnected systems often exhibit significant coupling between the various subsystems thus requiring an integrated approach to controller design. Short Take-Off and Landing (STOL) aircraft are examples of such systems. In STOL aircraft, the forces and moments generated by the propulsion system provide control and maneuvering capabilities for the aircraft at low speeds thus creating the need for Integrated Flight/Propulsion Control (IFPC) system design. On the other hand, there is the need to validate certain subsystem performance and robustness characteristics independently of the integrated system. This calls for a level of decentralization in control implementation.

One approach to integrated control design which combines aspects of centralized and decentralized control design approaches is currently being developed at NASA Lewis Research Center [ 1 ]. This approach consists of first designing a centralized controller, so that all subsystem interconnections are accounted for in the initial design stage, and then partitioning the centralized controller into separately implementable decentralized subcontrollers for individual subsystems. By partitioning here is meant representing the high-order centralized controller with two or more lower order subcontrollers which have input/output intercoupling such that the overall control law

obtained on assembling the subcontrollers closely approximates the input/output behavior of the centralized controller.

The objective of the current work is to describe a parameter optimization framework for obtaining partitioned subcontrollers with a decentralized hierarchical control structure. This structure is state-of-the-art in IFPC implementation and allows for separate implementation of airframe and propulsion control systems which, when assembled, will meet the overall design criteria for the integrated airframe/propulsion system.

The paper is organized as follows. The controller partitioning problem with the desired partitioning structure is stated first. A criterion for matching the performance of the centralized controller by partitioned subcontrollers while meeting the constraints on the partitioning structure 
is then described and a "cost function" is related to this criterion. Analytical expressions for the derivatives of the cost function with respect to the state-space parameters of the partitioned subcontrollers are developed. A parameter optimization algorithm using these analytic gradients to minimize the cost is then described. Finally, application of the algorithm to IFPC design using controller partitioning is discussed for a STOL aircraft in the short approach to landing task.

\section{Controller Partitioning and Related Cost}

The decentralized, hierarchical controller partitioning structure is shown in Fig. 1 with reference to an Integrated Flight/Propulsion Control system. In this figure, the subscripts and superscripts " $a$ " and " $e$ " refer to airframe and propulsion (engine) quantities, respectively, and the subscript " $c$ " refers to commanded quantities. The interface variables $\mathbf{z}_{e n}$ represent propulsion system quantities that affect the airframe, such as propulsive forces and moments. The structure is hierarchical in that the airframe (flight) controller produces commands for the interface variable $\left(\mathbf{z}_{e a_{c}}\right)$ which are tracked by the propulsion subsystem. The partitioning shown in Fig. 1 is simplified in that the controlled output errors and the interface variables $\mathbf{z}_{e a}$ are assumed to be the only inputs to the subcontrollers (e.g. there could also be direct feedbacks for stability augmentation). Another simplification made in the discussion in this paper is that the plant is assumed to have no direct feedthrough from control inputs, i.e. the plant " $D$ " matrix is zero. These assumptions maintain simplicity while conveying the basic idea behind the formulation and solution of the controller partitioning problem.

The controller partitioning problem can be stated as follows:

Given a centralized controller with transfer matrix $K(s)$ and a specification of the partitioning structure of controller inputs and outputs, i.e.

$$
\mathbf{u}=K(s) \mathbf{e} ; \quad \mathbf{u}=\left[\begin{array}{l}
\mathbf{u}_{a} \\
\mathbf{u}_{e}
\end{array}\right], \mathbf{e}=\left[\begin{array}{l}
\mathbf{e}_{a} \\
\mathbf{e}_{e}
\end{array}\right]
$$

where $\mathbf{u}_{a} \in R^{k_{a}}, \mathbf{u}_{e} \in R^{k_{e}}, \mathbf{e}_{a} \in R^{m_{a}}$, and $\mathbf{e}_{e} \in R^{m_{e}} ;$ a choice of interface variables $\mathbf{z}_{e a} \in R^{p_{e a}} ; \mathbf{a}$ plant with transfer matrix $\widehat{G(s)}$ of the form

$$
\widehat{G(s)}=\left[\begin{array}{c}
G(s) \\
G_{e a}(s)
\end{array}\right] \text { with }\left[\begin{array}{l}
\mathbf{z}_{a} \\
\mathbf{z}_{e}
\end{array}\right]=G(s) \mathbf{u} \text { and } \mathbf{z}_{e a}=G_{e a}(s) \mathbf{u}
$$


Find subcontrollers with stable transfer matrices $K^{a}(s)$ and $K^{e}(s)$, i.e.

$$
\left[\begin{array}{c}
\mathbf{u}_{a} \\
\mathbf{z}_{e a_{c}}
\end{array}\right]=K^{a}(s) \mathbf{e}_{a} \quad \text { and } \quad \mathbf{u}_{e}(s)=K^{e}(s)\left[\begin{array}{c}
\mathbf{e}_{e}(s) \\
\mathbf{e}_{e a}(s)
\end{array}\right]
$$

where $\mathbf{e}_{e a}=\mathbf{z}_{e a_{\varepsilon}}-\mathbf{z}_{e a}$, so that the closed-loop performance and robustness with the subcontrollers closely matches that with the centralized controller within the constraints of the partitioning structure.

The particular subsystem constraint for IFPC application is that the engine subcontroller $K^{e}(s)$ should have the structure of a command tracking controller for the interface variable commands $\mathbf{z}_{e a_{c}}$. Such a control structure allows the engine manufacturer to evaluate the engine subsystem performance independently of the airframe control and to verify that the engine subsystem will provide the desired performance when installed in the airframe. In general there will be practical constraints on the achievable bandwidth of $\mathbf{z}_{e a}$ tracking for the engine subcontroller. The lower bound on the $\mathbf{z}_{e a}$ command tracking bandwidth will be based on achieving the desired performance for the integrated system, while the upper bound will be imposed by actuator limits and robustness requirements to high frequency modeling uncertainties.

The approach considered in this paper for solving the controller partitioning problem is that of optimization of a suitable cost function over the state-space parameters of the partitioned subcontrollers using an analytical expression for the gradient of the cost function. Two requirements are essential for the success of such an optimization approach: (1) an initial choice of parameters (partitioned subcontrollers) and (2) a cost function which reflects the essence of the problem statement. The first requirement is met via a stepwise procedure for determining partitioned subcontrollers that meet the controller stability and the $\mathbf{z}_{e a}$ tracking structure and bandwidth requirements, as described in [2]. The subcontrollers obtained using this procedure are suitable for initializing the parameter optimization approach described herein. The cost function for controller partitioning is chosen to be of the form

$$
J(\mathbf{p})=J_{\mathbf{p e r r}}(\mathbf{p})+J_{\text {track }}(\mathbf{p})
$$


where $\mathbf{p}$ is the vector of parameters over which optimization takes place, $J_{\text {perr }}(\mathbf{p})$ reflects the performance requirements (including robustness) and $J_{\text {track }}(\mathbf{p})$ reflects the $\mathbf{z}_{e a}$ tracking requirement. The procedure for implementing the subcontroller stability constraint will be discussed later. In reference [ 3 ], a similar approach to controller partitioning was described; but, in that work, only the performance cost was used and a different cost function (the $H^{\infty}$ norm) was used to express it.

The performance cost $J_{\text {perf }}(\mathbf{p})$ is chosen to be

$$
J_{\text {perf }}=\int_{\omega_{1}}^{\omega_{2}} \sum_{i}\left(\sigma_{i}[(K(j \omega)-\widetilde{K(\mathbf{p})}(j \omega)) G(j \omega) W(j \omega)]\right)^{2} \mathrm{~d} \omega
$$

where $\widetilde{K}(s)$ is the "equivalent" centralized controller obtained on assembling the partitioned subcontrollers using appropriate plant information (the procedure for obtaining $\widetilde{K}(s)$ will be discussed later in the paper). The above choice of $J_{\text {perf }}(\mathbf{p})$ corresponds to matching the loop transfer function matrix at the control inputs, $\mathbf{u}$, during controller partitioning. Since the closed-loop performance and robustness are strictly a function of the loop transfer characteristics for the feedback structure being considered here, matching the loop transfer function matrix indirectly represents the requirement to match the centralized controller performance and robustness with the partitioned subcontrollers. The frequency band, $\left[\omega_{1}, \omega_{2}\right]$, is the interval over which a good match between loop transfer matrices is sought while the frequency dependent weighting matrix, $W$, allows for emphasizing certain frequency ranges and directions in obtaining this good approximation. In particular, choosing $W(j \omega)=(I+K(j \omega) G(j \omega))^{-1}$, the inverse of the return difference matrix, ensures that heavy emphasis will be placed on matching the closed-loop performance and robustness characteristics of the centralized controller by the partitioned controller.

Note that $J_{\text {perr }}(\mathbf{p})$ is the $H^{2}$ norm of the weighted difference between the loop transfer function matrices. We might have preferred on theoretical grounds to use the $H^{\infty}$ norm (cf., [ 5 ]), but this is numerically difficult to calculate and to differentiate in order to generate the gradient needed in the optimization process. 
The $\mathbf{z}_{e a}$ tracking cost $J_{\text {track }}(\mathbf{p})$ is chosen to be

$$
J_{\mathrm{track}}=\int_{\omega_{1}}^{\omega_{2}} \sum_{i} \lambda_{i}\left(\frac{\left\|T_{\text {cent }}^{i}(j \omega)-\widehat{T^{i}}(\mathbf{p})(j \omega)\right\|_{2}}{\left\|T^{i} \operatorname{cent}(j \omega)\right\|_{2}}\right)^{2} \mathrm{~d} \omega .
$$

$T_{\text {cent }}^{i}$ is the transfer function vector from the airframe commands $\mathbf{z}_{a_{c}}$ to the $i^{\text {th }}$ interface variable $\mathbf{z}_{e a}{ }^{i}$ with the centralized controller. $\widehat{T}^{i}$ is the transfer function vector from the airframe commands $\mathbf{z}_{a_{c}}$ to the $i^{\text {th }}$ interface variable as commanded by the partitioned airframe controller, $\mathbf{z}_{e a_{c}}{ }^{i}$, with the partitioned subcontrollers and $\lambda_{i}$ is a scalar weighting. $\|\cdot\|_{2}$ denotes the Euclidean norm of the row vector. Note that for the partitioned subcontrollers to closely match the performance achieved with the centralized controller, the response of the interface variables $\mathbf{z}_{\text {ea }}$ to airframe commands $\mathbf{z}_{a_{c}}$ with the partitioned subcontrollers must match the corresponding response with the centralized controller because the interface variables (e.g., propulsion system generated thrust) significantly affect the airframe responses $\mathbf{z}_{a}$. Thus requiring $T_{\text {cent }}^{i}$ to closely match $\widehat{T^{i}}$, as reflected in $J_{\text {track }}(\mathbf{p})$, will result in partitioned controllers such that $\mathbf{z}_{e a_{c}}{ }^{i}$ appears to be a command for $\mathbf{z}_{e a}{ }^{i}$. The scalars $\lambda_{i}$ are tuning parameters which allow the control designer to provide relative weighting for enforcing the command tracking structure among the various elements of $\mathbf{z}_{e a}^{i}$ and for trading off the performance cost against the cost of enforcing this command tracking structure. It is conjectured that the scalars $\lambda_{i}$ provide an indirect means for adjusting the $\mathbf{z}_{e a}{ }^{i}$ tracking bandwidth achieved by the optimized engine subcontroller. For instance, choosing $\lambda_{i}$ "large" will cause the optimization to proceed in a direction such that the $\mathbf{z}_{a_{c}} \rightarrow \mathbf{z}_{e a_{c}}{ }^{i}$ command generated by the airframe subcontroller "closely" matches the $\mathbf{z}_{a_{c}} \rightarrow \mathbf{z}_{e a}{ }^{i}$ response with the centralized controller. This observation together with the fact that the $\mathbf{z}_{a_{c}} \rightarrow \mathbf{z}_{e a}{ }^{i}$ response with the partitioned subcontrollers must closely match the corresponding response with the centralized controller in order to minimize $J_{\text {perf }}(\mathbf{p})$ implies that the optimized subcontrollers will be such that $\mathbf{z}_{e a_{c}}{ }^{i}$ and $\mathbf{z}_{e a}{ }^{i}$ match very closely. This in turn implies that the optimized engine subcontroller will be such that the engine subsystem has "large" tracking bandwidth for the $\mathbf{z}_{e a}{ }^{i}$ command.

\section{Cost Function and Gradient Evaluation}

Cost Function. The parameters in the optimization process are certain entries in state-space 
realizations of $K^{a}(s)$ and $K^{e}(s)$ as defined in the following. The notation $M_{o i}^{s}$ is used throughout to indicate the matrix $M \in\{A, B, C, D\}$ in the state-space realization of the system transfer matrix $s \in\{c, p, a, e\}$ ( $c=$ centralized controller, $p=$ plant, $a=$ airframe subcontroller, $e=$ engine subcontroller) with input $i$ (resp. output o) $\in\left\{p, a, e, e a_{c}, e a\right\}$

$$
\begin{aligned}
& K^{a}(s): \quad\left[\begin{array}{c}
\mathbf{u}_{a} \\
\mathbf{z}_{e a_{c}}
\end{array}\right]=\left(\left(\begin{array}{c}
C_{a a}^{a} \\
C_{e a_{c} a}^{a}
\end{array}\right)\left(s I-A^{a}\right)^{-1} B_{a a}^{a}+\left(\begin{array}{c}
D_{a a}^{a} \\
D_{e a_{c} a}^{a}
\end{array}\right)\right) \mathbf{e}_{a} \\
& K^{e}(s): \quad \mathbf{u}_{e}=\left(C_{e e}^{e}\left(s I-A^{e}\right)^{-1}\left(B_{e e a}^{e} B_{e e}^{e}\right)+\left(D_{e e a}^{e} D_{e e}^{e}\right)\right)\left[\begin{array}{c}
\mathbf{e}_{e a} \\
\mathbf{e}_{e}
\end{array}\right] .
\end{aligned}
$$

One consideration in choosing parameterizations is to introduce a "minimal" number of parameters in the optimization process. A form for the state-space realizations introduced in [ 6 ] was used as the model for our parameterizations. The subcontroller system matrices $A^{a}$ and $A^{e}$ are represented as block diagonal matrices with two-by-two real companion blocks of the form $\left[\begin{array}{ll}0 & 1 \\ \alpha & \beta\end{array}\right]$. Note that, if the order of either $A^{a}$ or $A^{e}$ is odd, there is also one diagonal real entry corresponding to a real eigenvalue. In addition, $\alpha$ and $\beta$ are constrained to be negative in order to meet the requirement that subcontrollers be stable. In addition the first columns of each of the subcontroller input matrices $B_{a a}^{a}$ and $B^{e}=\left[\begin{array}{ll}B_{e e a}^{e} & B_{e e}^{e}\end{array}\right]$ are fixed at non-zero values given by the initial partitioning. This last condition is different from the one used in [6] where the first column of the $B$ matrix was fixed as the unit vector $\mathbf{e}_{1}$. The initial partitioning for the numerical example considered in this paper could not be put into this latter form necessitating the use of the alternative form. This alternate form is discussed in reference [ 4 ].

The parameters over which the optimization takes place are then the $\alpha$ and $\beta$ entries in the block canonical forms, the entries in all but the first columns of the matrices $B_{a a}^{a}$ and $B^{e}$ and all the entries in the matrices $\left[\begin{array}{c}C_{a a}^{a} \\ C_{e a_{c} a}^{a}\end{array}\right], C_{e e}^{e},\left[\begin{array}{c}D_{a a}^{a} \\ D_{e a_{c} a}^{a}\end{array}\right]$ and $\left[D_{e e a}^{e} D_{e e}^{e}\right]$. The parameter vector will be denoted as $\mathbf{p} \in R^{N}$ where $N=n_{a}\left(k_{a}+m_{a}+p_{e a}\right)+k_{a}\left(m_{a}+p_{e a}\right)+n_{e}\left(k_{e}+m_{e}+p_{e a}\right)+\left(k_{e}+p_{e a}\right) m_{e}$. The number of parameters depends not only on the total numbers of controller inputs and outputs and interface variables which are fixed but also on the orders of the subcontrollers, $n_{a}$ and $n_{e}$. There is thus a double incentive for keeping these orders low; not only to reduce the complexities of the subcontrollers but also to accelerate the optimization algorithm whose performance depends 
on the number of parameters.

State-space representations for the transfer matrices $K(s)$ and $\widehat{G(s)}=\left[\begin{array}{c}G(s) \\ G_{e a}(s)\end{array}\right]$ are given while those for $\widetilde{K}(s), T_{\text {cent }}(s)$ and $\widehat{T}(s)$ are constructed from the state-space representations of $K(s), K^{a}(s), K^{e}(s), G_{e a}(s)$ and $G(s)$.

The transfer matrix $\widetilde{K}(s)$ which enters into the performance cost term $J_{\text {perr }}(\mathbf{p})$ depends on $K^{a}(s)$ and $K^{e}(s)$, and on the transfer submatrix of the plant from control inputs (u) to interface variables $\left(\mathbf{z}_{e a}\right), G_{e a}: \quad \mathbf{z}_{e a}=C_{e a p}^{p}\left(s I-A^{p}\right)^{-1}\left[\begin{array}{ll}B_{p a}^{p} & B_{p e}^{p}\end{array}\right]\left[\begin{array}{l}\mathbf{u}_{a} \\ \mathbf{u}_{e}\end{array}\right]$. A state-space realization for the assembled controller $\widetilde{K}(s)=\widetilde{C}(s I-\tilde{A})^{-1} \widetilde{B}+\widetilde{D}$ was shown in [3] to be

$$
\begin{aligned}
& \tilde{A}=\left[\begin{array}{ccc}
\left(A^{p}-B_{p e}^{p} D_{e e a}^{e} C_{e a p}^{p}\right) & \left(B_{p a}^{p} C_{a a}^{a}+B_{p e}^{p} D_{e e a}^{e} C_{e a_{c} a}^{a}\right) & B_{p e}^{p} C_{e e}^{e} \\
0 & A^{a} & 0 \\
-B_{e e a}^{e} C_{e a p}^{p} & B_{e e a}^{e} C_{e a_{c} a}^{a} & A^{e}
\end{array}\right] \\
& \widetilde{B}=\left[\begin{array}{cc}
\left(B_{p a}^{p} D_{a a}^{a}+B_{p e}^{p} D_{e e a}^{e} D_{e a_{c} a}^{a}\right) & B_{p e}^{p} D_{e e}^{e} \\
B_{a a}^{a} & 0 \\
B_{e e a}^{e} D_{e a_{c} a}^{a} & B_{e e}^{e}
\end{array}\right] \\
& \widetilde{C}=\left[\begin{array}{ccc}
0 & C_{a a}^{a} & 0 \\
-D_{e e a}^{e} C_{e a p}^{p} & D_{e e a}^{e} C_{e a_{c} a}^{a} & C_{e e}^{e}
\end{array}\right] \text { and } \\
& \widetilde{D}=\left[\begin{array}{cc}
D_{a a}^{a} & 0 \\
D_{e e a}^{e} D_{e a_{c} a}^{a} & D_{e e}^{e}
\end{array}\right]
\end{aligned}
$$

The state-space representation for $T_{\text {cent }}(s)=C^{\text {cent }}\left(s I-A^{\text {cent }}\right)^{-1} B^{\text {cent }}+D^{\text {cent }}$ whose rows enter into the evaluation of the cost term $J_{\text {track }}(\mathbf{p})$ can be written in terms of the submatrices in the state-space representations for $K(s)$ and $G(s)$, i.e. $K(s)=\left[\begin{array}{c}C_{a c}^{c} \\ C_{e c}^{c}\end{array}\right]\left(s I-A^{c}\right)^{-1}\left[\begin{array}{ll}B_{c a}^{c} & B_{c e}^{c}\end{array}\right]+\left[\begin{array}{c}D_{a a}^{c} \\ D_{e e}^{c}\end{array}\right]$ and $G(s)=\left[\begin{array}{l}C_{a p}^{p} \\ C_{e p}^{p}\end{array}\right]\left(s I-A^{p}\right)^{-1}\left[\begin{array}{ll}B_{p a}^{p} & B_{p e}^{p}\end{array}\right]$. As is shown in $[4]$,

$$
\begin{aligned}
A^{\text {cent }} & =\left[\begin{array}{cc}
A^{c} & \left(-B_{c a}^{c} C_{a p}^{p}-B_{c e}^{c} C_{e p}^{p}\right) \\
\left(B_{p a}^{p} C_{a c}^{c}+B_{p e}^{p} C_{e c}^{c}\right) & A^{p}-B_{p a}^{p} D_{a a}^{c} C_{a p}^{p}-B_{p e}^{p} D_{e e}^{c} C_{e p}^{p}
\end{array}\right] \\
B^{\text {cent }} & =\left[\begin{array}{c}
B_{c a}^{c} \\
B_{p a}^{p} D_{a a}^{c}
\end{array}\right] \quad C^{\text {cent }}=\left[\begin{array}{lll}
0 C_{e a p}^{p}
\end{array}\right] \quad D^{\text {cent }}=0 .
\end{aligned}
$$

Similarly, $\widehat{T}(s)=\widehat{C}(s I-\widehat{A})^{-1} \widehat{B}+\widehat{D}$ whose rows also appear in $J_{\text {track }}(\mathbf{p})$ can be written in 
terms of the state-space representations for $K^{a}(s), K^{e}(s)$ and $G(s)$

$$
\begin{aligned}
& \widehat{A}=\left[\begin{array}{ccc}
A^{a} & 0 & -B_{a a}^{a} C_{a p}^{p} \\
B_{e e a}^{e} C_{e a_{c} a}^{a} & A^{e} & \widehat{A}_{e p} \\
\widehat{A}_{p a} & B_{p e}^{p} C_{e e}^{e} & \widehat{A}_{p p}
\end{array}\right] \\
& \widehat{B}=\left[\begin{array}{c}
B_{a a}^{a} \\
B_{e e a}^{e} D_{e a_{c} a}^{a} \\
\left(B_{p a}^{p} D_{a a}^{a}+B_{p e}^{p} D_{e e a}^{e} D_{e a_{c} a}^{a}\right)
\end{array}\right] \\
& \widehat{C}=\left[\begin{array}{lll}
C_{e e a}^{e} & 0 & -\left(D_{e a_{c} a}^{a} C_{a p}^{p}\right)
\end{array}\right] \text { and } \hat{D}=D_{e a_{c} a}^{a}
\end{aligned}
$$

where $\hat{A}_{e p}=-\left(B_{e e}^{e} C_{e p}^{p}+B_{e e a}^{e} C_{e a p}^{p}+B_{e e a}^{e} D_{e a_{a} a}^{a} C_{a p}^{p}\right), \hat{A}_{p a}=\left(B_{p a}^{p} C_{a a}^{a}+B_{p e}^{p} D_{e e a}^{e} C_{e a_{c} a}^{a}\right)$ and $\hat{A}_{p p}=$ $\left(A^{p}-B_{p a}^{p} D_{a a}^{a} C_{a p}^{p}-B_{p a}^{p} D_{e e a}^{e} D_{e a_{a} a}^{a} C_{a p}^{p}-B_{p e}^{p} D_{e e a}^{e} C_{e a p}^{p}-B_{p e}^{p} D_{e e}^{e} C_{e p}^{p}\right)$.

The total cost function is calculated by applying Simpson's Rule for numerical integration over the frequency interval $\left[\omega_{1}, \omega_{2}\right]$ to the sum of the two terms $J_{\text {perf }}(\mathbf{p})$ and $J_{\text {track }}(\mathbf{p})$ described in formulas (1) and (2) respectively. The expressions developed above for the state-space representations of $K(s), \widetilde{K}(s), T_{\text {cent }}(s)$ and $\widehat{T}(s)$ are used for calculating these costs. Note that $\lambda_{i}$ in $J_{\text {track }}(\mathbf{p})$ are to be chosen independently by the control designer to achieve the desired tracking bandwidth for $\mathbf{z}_{e a}{ }^{i}$.

Analytic Gradient Development. In addition to requiring the values of the "combined cost function," $J(\mathbf{p})$, the optimization method which is used requires the gradient $\nabla J(\mathbf{p})$ which, for any specific parameter vector $\mathbf{p}$, is a vector with $N$ entries consisting of the partial derivatives of $J$ with respect to each parameter. The calculation of these partial derivatives for the $J_{\text {perf }}(\mathbf{p})$ portion of the cost function is illustrated in the following. A similar procedure is used to calculate the partial derivatives of $J_{\text {track }}(\mathbf{p})$. Complete expressions for the partial derivatives are available in [4].

A formula for the partial derivatives of distinct non-zero singular values, $\sigma_{i}[A]$, with respect to an element $\rho$ of the parameter vector $\mathbf{p}$ which can be found in reference [ 7 ] is

$$
\frac{\partial \sigma_{i}[A(\mathbf{p})]}{\partial \rho}=\operatorname{Re}\left[\mathbf{u}_{i}^{*} \frac{\partial A(\mathbf{p})}{\partial \rho} \mathbf{v}_{i}\right]
$$

where $\mathbf{u}_{i}$ and $\mathbf{v}_{i}$ are right and left normalized singular vectors of $A$ and where the superscript * denotes the conjugate transpose. 
For any specific parameter $\rho$ (i.e. component of $\mathbf{p}$ ), the partial derivative of $J_{\text {perf }}(\mathbf{p})$ with respect to $\rho$ may be expressed as

$$
\begin{aligned}
\frac{\partial J_{\text {perr }}(\mathbf{p})}{\partial \rho} & =\frac{\partial\left[\int_{\omega_{1}}^{\omega_{2}} \sum_{i}\left(\sigma_{i}[H(\omega, \mathbf{p})]\right)^{2} \mathrm{~d} \omega\right]}{\partial \rho} \\
& =\int_{\omega_{1}}^{\omega_{2}} \sum_{i} 2 \sigma_{i} \operatorname{Re}\left[\mathbf{u}_{i}^{*} \frac{\partial H(\omega, \mathbf{p})}{\partial \rho} \mathbf{v}_{i}\right] \mathrm{d} \omega
\end{aligned}
$$

where $H(\omega, \mathbf{p})=(K(j \omega)-\widetilde{K}(\mathbf{p})(j \omega)) G(j \omega) W(j \omega)$.

The partial derivatives of $H(\omega, \mathbf{p})$ with respect to each of the parameters $\rho$ of $\mathbf{p}$ are derived by noting that the only terms involving $\mathbf{p}$ in $H(\omega, \mathbf{p})$ arise from the $\widetilde{K}(\mathbf{p})(j \omega)$ factor. Using the "product rule" to differentiate the state space representation, $\widetilde{K}(\mathbf{p})(j \omega)=\widetilde{C}(j \omega I-\widetilde{A})^{-1} \widetilde{B}+\widetilde{D}$, and then multiplying from the right by $G(j \omega) W(j \omega)$ yields

$$
\begin{aligned}
& \frac{\partial H(\omega, \mathbf{p})}{\partial \rho}=-\frac{\partial \widetilde{C}(\mathbf{p})}{\partial \rho}(j \omega I-\widetilde{A})^{-1} \widetilde{B} G(j \omega) W(j \omega) \\
&-\widetilde{C}(j \omega I-\widetilde{A}(\mathbf{p}))^{-1} \frac{\partial \widetilde{A}(\mathbf{p})}{\partial \rho}(j \omega I-\widetilde{A}(\mathbf{p}))^{-1} \widetilde{B} G(j \omega) W(j \omega) \\
& \quad-\widetilde{C}(j \omega I-\tilde{A})^{-1} \frac{\partial \widetilde{B}(\mathbf{p})}{\partial \rho} G(j \omega) W(j \omega)-\frac{\partial \widetilde{D}(\mathbf{p})}{\partial \rho} G(j \omega) W(j \omega) .
\end{aligned}
$$

Substituting (4) in (3) yields an expression for $\frac{\partial J_{\text {perr }}(\mathbf{p})}{\partial \rho}$.

The final step needed to carry out the determination of the gradient of $J_{\text {perf }}$ is to calculate the partial derivatives of $\widetilde{A}, \widetilde{B}, \widetilde{C}$, and $\widetilde{D}$ with respect to the parameters which appear in these matrices via the subcontroller state-space matrices

$$
M \in\left\{A^{a}, B_{a a}^{a}, C_{a a}^{a}, C_{e a_{c} a}^{a}, D_{a a}^{a}, D_{e a_{c} a}^{a}, A^{e}, B_{e e}^{e}, B_{e e a}^{e}, C_{e e}^{e}, D_{e e a}^{e} D_{e e}^{e}\right\}
$$

as described earlier. Each parameter $\rho$ corresponds to a specific row and column element of some parameter submatrix, say the element in row $j$, column $k$ of submatrix $M$. Any block of $\widetilde{A}, \widetilde{B}$, etc. not containing $M$ as a factor will be replaced by a zero block in the calculation of the corresponding 
partials $\frac{\partial \widetilde{A}}{\partial \rho}, \frac{\partial \widetilde{B}}{\partial \rho}$, etc. On the other hand, any block containing $M$ as a factor contributes the same block with the factor $M$ replaced by the submatrix $\mathbf{e}_{j} \mathbf{e}_{k}^{\top}$, which has the $j, k^{\text {th }}$ - entry as its only nonzero element. Here $\mathbf{e}_{j}$ is the vector whose $j^{\text {th }}$ element is 1 and all other elements are zero. For example if $\rho$ corresponds to the row $j$, column $k$ entry of $A^{a}$, then

$$
\frac{\partial \tilde{A}}{\partial \rho}=\left[\begin{array}{ccc}
0 & 0 & 0 \\
0 & \mathbf{e}_{j} \mathbf{e}_{k}^{\top} & 0 \\
0 & 0 & 0
\end{array}\right]
$$

while $\frac{\partial \widetilde{B}}{\partial \rho}, \frac{\partial \widetilde{C}}{\partial \rho}$ and $\frac{\partial \widetilde{D}}{\partial \rho}$ are all 0 . If $\rho$ corresponds to the row $j$, column $k$ entry of $D_{e e a}^{e}$, then

$$
\frac{\partial \widetilde{A}(\mathbf{p})}{\partial \rho}=\left[\begin{array}{ccc}
-B_{p e}^{p} \mathbf{e}_{j} \mathbf{e}_{k}^{\top} C^{p} & B_{p e}^{p} \mathbf{e}_{j} \mathbf{e}_{k}^{\top} C_{\mathrm{ea}_{c} a}^{a} & 0 \\
0 & 0 & 0 \\
0 & 0 & 0
\end{array}\right]
$$

with expressions for $\frac{\partial \widetilde{B}(\mathbf{p})}{\partial \rho}, \frac{\partial \widetilde{C}(\mathbf{p})}{\partial \rho}$, and $\frac{\partial \widetilde{D}(\mathbf{p})}{\partial \rho}$ derived in a similar manner. Here the 0 entries refer to zero blocks. These two examples demonstrate extremes of complexity in the calculation of the various partials of the state space matrices for $\widetilde{K}(s)$. Once these partials have been determined with respect to all parameters in $\mathbf{p}$, the resulting matrices can be inserted into the formula for the partials of $J_{\text {perr }}$ described earlier and the gradient can be calculated by applying Simpson's Rule of numerical integration over the same frequency interval as was used for evaluating $J_{\text {perr }}(\mathbf{p})$.

The partial derivatives of $J_{\text {irack }}$ are computed by a similar procedure and these are combined with the partials of $J_{\text {perr }}(\mathbf{p})$ to yield $\nabla J(\mathbf{p})=\nabla J_{\text {perr }}(\mathbf{p})+\nabla J_{\text {track }}(\mathbf{p})$.

\section{The Optimization Algorithm}

The flow of the optimization algorithm for controller partitioning is shown in Fig. 2.

The fixed data used by the algorithm are state-space representations for the plant submatrices $G(s)$ and $G_{e a}(s)$, the centralized controller $K(s)$, the weighting matrix $W(s)$, as well as a partitioning structure for the controller inputs, outputs and interface variables. The control designer must also input values of the tracking weight parameters $\lambda_{i}$ used to determine $J_{\text {track }}(\mathbf{p})$. The main steps in the algorithm are: 
1. The initial partitioning $\left(K_{0}^{a}(s)\right.$ and $\left.K_{0}^{e}(s)\right)$ is obtained by applying the stepwise procedure described in [ 2 ]. Special attention is paid to obtaining reasonably low-order subcontrollers which are stable and satisfy the $\mathbf{z}_{\mathfrak{e a}}$ command-tracking requirement.

2. The initial partitioning is converted to the "minimal parameter" form to generate an initial value of the parameter vector.

3. The (initial as well as any subsequent value of the) parameter vector is passed to a module which determines the state-space representation for the hierarchically partitioned controller and calculates the combined cost. The gradient is also computed analytically by the procedure described above.

4. The current gradient is used in conjunction with previous information to generate a direction of search. A one-dimensional linesearch is carried out using the cost and gradient calculated at each parameter point to predict a new parameter point until one is found which yields a sufficient reduction in both the cost function and the size of the gradient. This linesearch is constrained so as to maintain stability of the subcontrollers.

5. At the end of the linesearch, the new parameter point, cost and gradient are compared to the values at the beginning of the linesearch as a check on convergence. If either the maximum absolute value of the partial derivatives is less than a user-specified tolerance or both the maximum change in all the parameters is less than one user-specified value and the change in the total cost is less than another value then convergence is declared and the iteration ceases. If the convergence test fails, the algorithm proceeds to update the information used to determine the direction of search and to use the most recent cost and gradient values to generate a new direction of search and carry out the linesearch via steps 3 . and 4 .

6. The output of the algorithm is the state-space representation for subcontrollers which yield the assembled hierarchically partitioned controller with performance and stability robustness comparable (within convergence criteria) to that of the centralized controller and with reasonable adherence to the $z_{e n}$ command-tracking requirement. 
7. These subcontroller transfer matrices have the same orders $n_{a}$ and $n_{e}$ as the initial partitioning. Controller reduction can be performed on these "optimal subcontrollers" and the process of optimization can be repeated on the "new initial partitioning".

We use the Broyden Fletcher Goldfarb Shanno implementation of the quasi-Newton method to carry out this optimization, see [ 8 ]. This iterative method requires the calculation of the combined cost and gradient for parameters as referred to above. It uses successive gradients to build up an approximation to the inverse Hessian matrix. In this way, subsequent search steps are successively closer to those generated by Newton's Method and convergence is accelerated as the iterations proceed.

The results of applying this process to an IFPC example are reported in the following section.

\section{Example of Controller Partitioning}

The controller partitioning algorithm was applied to the centralized flight/propulsion controller for a STOL aircraft obtained in reference [ 9 ]. This controller has the form $\mathbf{u}=K(s)$ e with the error vector e consisting of errors, $\mathbf{e}=\left[e_{q}, e_{v}, e_{N 2}, e_{E P R}\right]^{\top}$, in following pitch rate variable $\left(q_{v}=q+0.1 \theta\right)$, velocity $(v)$, engine fan speed $(N 2)$ and engine pressure ratio $(E P R)$ commands. The control input vector $\mathbf{u}$ consists of rates of change of thrust vectoring angle, fuel flow, thrust reverser port area and nozzle throat area, $\mathbf{u}=\left[\dot{\delta}_{T V}, \dot{W F}, \dot{A 78}, \dot{A 8}\right]$. Note that $\mathbf{u}$ consists of rates because integrators were appended to the control inputs during the process of centralized control design to achieve zero steady-state error for step commands. Based on open loop control effectiveness studies for the plant, the partitioned airframe and engine controllers are desired to have inputs $\mathbf{e}_{a}=\left[e_{q}, e_{V}\right]^{\top}$ and $\mathbf{e}_{e}=\left[e_{N 2}, e_{E P R}\right]^{\top}$ and outputs $\mathbf{u}_{a}=\left[\delta_{T V} \dot{ }\right]$ and $\mathbf{u}_{e}=[\dot{W} F, \dot{A 78}, \dot{A 8}]^{\top}$ respectively. The integrated plant and the centralized controller state-space matrices are listed in the Appendix. The interface variable $\mathbf{z}_{e a}$ for this design example is FEX, the axial thrust generated by the propulsion system, since the main function of the propulsion system is to provide the necessary axial thrust required to track pitch rate and velocity commands. The structure of this controller partitioning is shown in Fig. 3. 
An initial controller partitioning was obtained using the procedure discussed in [ 2 ]. The plant, centralized controller, and initial partitioning state-space matrices are listed in the Appendix. The airframe and engine subcontrollers for this initial partitioning are of order 10 and 7 , respectively. The performance of the initial controller partitioning was evaluated in comparison with that of the centralized controller by comparing closed-loop system response to step commands in the controlled variables $\mathbf{z}$. The responses to $q_{v_{c}}, N 2_{c}$ and $E P R_{c}$ with the initial partitioned subcontrollers were comparable to those with the centralized controller. However, the response to $V_{c}$, shown in Fig. 4, showed considerable degradation in terms of increased coupling in the $N 2$ and $E P R$ responses with the partitioned subcontrollers. Note that all the quantities shown in Fig. 4 were normalized, using scalings discussed in [ 9 ], to allow a direct comparison of the various response magnitudes.

For all the results presented in this paper, the optimization was done over the frequency range $\omega \in[0.1,100]$ with 20 frequency points per decade. The number of parameters in the optimization was 95 with 44 corresponding to the airframe subcontroller and 51 corresponding to the engine subcontroller. The algorithm was first applied with $\lambda=0\left(p_{e a}=1\right.$, so $\left.\lambda_{i}=\lambda\right)$, i.e. $J(\mathbf{p})=J_{\text {perr }}(\mathbf{p})$ with no attention to the $\mathbf{z}_{e a_{c}}$ command tracking structure requirement for the engine subcontroller. As seen from Fig. 5, the optimization code showed good numerical behavior with convergence to a significantly reduced cost $J(\mathbf{p})$ in 100 iterations. The maximum singular value of the weighted loop transfer error $\sigma_{\max }\left[(K-\widetilde{K}) G(I+K G)^{-1}\right](j \omega)$, which is a major contributor to the cost $J(\mathbf{p})$ in this case, shows a considerable improvement with partitioning optimization as is seen from Fig. 6. The results in Fig. 6 imply that the optimized partitioned controllers will more closely match the performance and robustness characteristics of the centralized controller than will the initial partitioned controllers. The closed-loop response to step $V_{c}$ with the optimized partitioned controllers is shown in Fig. 4 and is comparable to that for the centralized controller both in terms of tracking of the velocity command and decoupling of $q_{v}, N 2$ and $E P R$ responses. The closed loop responses with the optimized partitioned controllers for step $q_{v_{c}}, N 2_{c}$ and $E P R_{c}$ were also checked to ensure that these were comparable to the responses with the centralized controller.

Figure 7 shows the Bode gain plots for the interface variable command tracking response 
$\left(\left|F E X / F E X_{c}\right|\right)$ for the engine subsystem with the initial partitioning and the optimized partitioning obtained using $\lambda=0$. The engine subcontroller for this optimized partitioning has a $F E X$ command tracking bandwidth of approximately $4 \mathrm{rad} / \mathrm{s}$ (tracking bandwidth is defined as the frequency at which the Bode gain is $-3 \mathrm{~dB}$ ), which is quite low and implies that the engine subsystem will not provide adequate tracking of $F E X_{c}$ in the presence of disturbances and low frequency variations in the engine dynamics. In order to verify command tracking and disturbance rejection properties of the engine subsystem in independent subsystem check-out, it is desirable to have a minimum bandwidth of around $10 \mathrm{rad} / \mathrm{s}$ for $F E X$ command tracking. Since $F E X$ has significant control effect on the aircraft responses $V$ and $q_{v}$, such a lower bound on $F E X$ command tracking is also required to guarantee performance robustness of the integrated system in terms of tracking and decoupling of responses to $V$ and $q_{v}$ commands. As seen from Fig. 7, the initial controller partitioning satisfies this $F E X$ command tracking bandwidth.

The fact that the optimized partitioning with $\lambda=0$ gives performance that is comparable to the performance achieved with the centralized controller although the optimized engine subcontroller has poor $F E X$ command tracking properties can be understood by analysis of the results shown in Fig. 8. We note that the $F E X$ response to $V_{c}$ with the optimized $(\lambda=0)$ partitioned subcontrollers closely matches the corresponding response with the centralized controller. This result is as expected because $F E X$ significantly affects the $V$ and $q_{v}$ response of the aircraft and matching the $F E X$ response will be required if the partitioned subcontrollers are to match the performance achieved with the centralized controller. However, as seen from Fig. 8, the command for the interface variable $F E X_{c}$ as generated by the partitioned airframe subcontroller significantly "leads" the actual $F E X$ response. So, the optimized airframe subcontroller compensates for the low interface variable command tracking bandwidth property of the engine subcontroller by generating a command for the interface variable which leads the desired interface variable response by an appropriate amount. This phenomenon of lead compensation for low tracking bandwidth is further evident from the Bode gain plots shown in Fig. 9 for the $F E X / V_{c}$ response with the centralized controller and the $F E X / F E \dot{X}_{c}, F E X_{c} / V$ and $F E X / V_{c}$ responses with the optimized controller 
partitioning $(\lambda=0)$. In Fig. 9, the Bode gains of $F E X_{c} / V_{c}$ and $F E X / F E X_{c}$ do not exactly add up to the Bode gains of $F E X / V_{c}$ for the partitioned subcontrollers because the $F E X / F E X_{c}$ response is only for the engine subsystem and does not account for the effect of thrust vectoring $\left(\mathbf{u}_{a}\right)$ on the $F E X$ response.

As previously discussed, there is a minimum $\mathbf{z}_{e a}$ command tracking bandwidth which must be provided by the partitioned engine subcontroller for independent validation of the engine subsystem and performance robustness of the integrated system, and there is an upper limit on the achievable bandwidth imposed by actuator and stability robustness constraints. Some "lead" in the interface variable command $\mathbf{z}_{e a_{c}}$ generated by the airframe subcontroller is desirable to compensate for the limited bandwidth engine subsystem. Nevertheless, large "lead" is undesirable as it can result in quick saturation of the engine actuators because of command magnification. The effect of varying the user specified weights $\left(\lambda_{i}\right.$ in equation (2)) in the controller partitioning optimization procedure to address the trade-off between lead and bandwidth is demonstrated by the plots of the Bode gains of the $F E X / F E X_{c}$ responses shown in Fig. 7. As is seen there, the $F E X$ command tracking bandwidth achieved by the optimized engine subcontroller increased as $\lambda$ was increased. The closedloop system performance of the optimized partitioning with $\lambda=0.05$ for step $V_{c}$ closely matched the performance with the centralized controller as shown in Fig. 10 (note that the scales in Fig. 10 are different from those in Fig. 4). The airframe subcontroller obtained from the $\lambda=0.05$ optimal partitioning also provided small lead in the $F E X$ command, as is seen from Fig. 11, and the $F E X$ command tracking bandwidth of $20 \mathrm{rad} / \mathrm{s}$ (from Fig. 7) for the corresponding engine subcontroller was within the engine actuation limits. The state-space matrices for these airframe and engine subcontrollers are listed in the Appendix.

With the optimized controller partitioning for $\lambda=0.05$ as a baseline, the possibility of reducing the order of the partitioned subcontrollers was investigated. The engine subcontroller was reduced to $4^{\text {th }}$ order by residualization of the three high frequency modes without any loss of performance. Through the use of internally balanced reduction techniques [ 10 ], the airframe subcontroller was reduced to $6^{\text {th }}$ order (from the original $10^{\text {th }}$ order) without excessive mismatch in the controller 
transfer matrix characteristics as is seen from the full and reduced order airframe controller singular values comparison in Fig. 12. This reduced order airframe subcontroller did, however, exhibit deterioration in closed-loop performance as shown in the $V$ and $q_{v}$ response comparison plots for step $V_{c}$ in Fig. 13. The controller partitioning optimization procedure was performed with $\lambda=0.05$ and with optimization over only the airframe subcontroller parameters (the engine subcontroller was fixed to the reduced $4^{\text {th }}$ order controller). The response obtained with the optimized reduced order airframe subcontroller for step $V_{c}$ is also shown in Fig. 13. Note that the optimized subcontroller provides improved tracking of the velocity command. Although, as can be seen from Fig. 13, the transient in $q_{v}$ is larger in magnitude for the optimized reduced order subcontroller than for the initial reduced order airframe subcontroller, the transient is of significantly shorter duration and the pitch variable quickly settles down to the zero commanded value. The state-space matrices for the optimized reduced order subcontrollers are listed in the Appendix. These results demonstrate the potential for using the controller partitioning procedure in conjunction with controller order reduction techniques to obtain the simplest (lowest order) partitioned subcontrollers that will provide adequate integrated system performance.

The results presented so far have focused on comparing the performance achieved with the optimized partitioned subcontrollers with that achieved with the centralized controller. Robustness issues are also of importance in practical control design. Robustness analysis was performed using structured singular values for gain and phase variations occuring in the controlled outputs. The results are shown in Fig. 14 for the centralized controller, the optimized full order partitioned subcontrollers corresponding to $\lambda=0.05$ and the optimized reduced order subcontrollers. The procedure for creating the interconnection matrix to perform gain and phase margin robustness analysis using structured singular values is documented in Ref. [ 11 ] and other references listed therein. From Fig. 14 the stability margin parameter $\mu$ corresponding to the maximum over frequency of the structured singular values is 1.37 for the centralized controller and 1.43 for the full and reduced order optimized partitioned subcontrollers. These values of $\mu$ translate into guaranteed multivariable gain margins of $-4.8 \mathrm{~dB}$ to $11.4 \mathrm{~dB}$ and $-4.6 \mathrm{~dB}$ to 10.5 for the closed-loop system with the cen- 
tralized and partitioned subcontrollers respectively, and similarly guaranteed multivariable phase margins of \pm 42.8 and $\pm 41.0 \mathrm{deg}$, respectively, for simultaneous gain or phase variations occuring in all loops at the controlled outputs. These results indicate that the robustness characteristics of the centralized controller are maintained by application of the parameter optimization approach.

\section{Conclusions}

A parameter optimization framework was presented to solve the problem of partitioning a centralized controller into a decentralized, hierarchical structure suitable for integrated flight/ propulsion control implementation. A cost function to be minimized was formulated such that the "equivalent" centralized controller assembled from the "optimized" partitioned subsystem controllers will closely match the performance and robustness of the closed-loop system with the centralized controller while maintaining the desired characteristics of the controller partitioning structure. An analytical gradient based optimization algorithm was developed and applied to controller partitioning of an integrated flight/propulsion control design for a modern fighter aircraft in the longitudinal short approach to landing task. The optimization of the partitioned subcontroller state-space parameters resulted in improved performance over an initial partitioning and close matching of the performance with the centralized controller. The ability of the optimization to address the subsystem control structure requirement of an adequate command tracking bandwidth for the propulsion subsystem by varying scalar weight parameters in the cost function was demonstrated for the example study. It was shown that by judicious use of the partitioning optimization procedure in conjunction with model reduction techniques, simplified low-order subsystem controllers with a desired structure can be obtained that match the performance and robustness characteristics of a high-order centralized controller. 


\section{APPENDIX}

The data matrices and initial partitioning matrices for the controller partitioning example are listed below. In all cases the given matrices $A, B, C$ and $D$ correspond to the state-space representation of the given system or subsystem

$$
\begin{aligned}
\frac{\mathrm{d} \mathbf{x}}{\mathrm{d} t} & =A \mathbf{x}+B \mathbf{y} \\
\mathbf{u} & =C \mathbf{x}+D \mathbf{y} .
\end{aligned}
$$

These matrices will be given in the form

$$
\left[\begin{array}{l|l}
A & B \\
\hline C & D
\end{array}\right]
$$

if space allows or will be written out individually if the former is too large.

The integrated airframe propulsion system with integrator augmentation is represented by

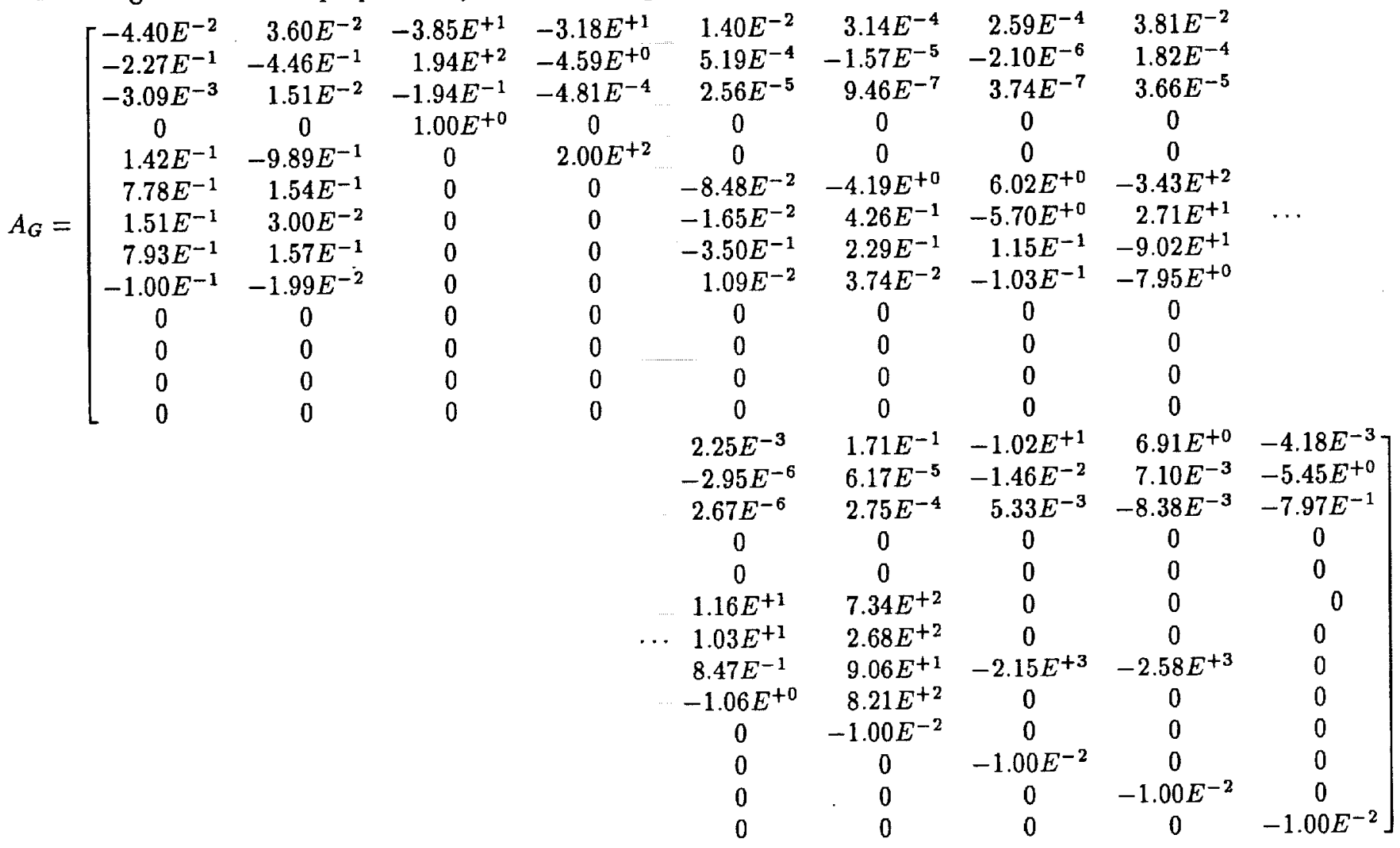

$$
\begin{aligned}
& B_{G}=\left[\begin{array}{cccc}
0 & 0 & 0 & 0 \\
0 & 0 & 0 & 0 \\
0 & 0 & 0 & 0 \\
0 & 0 & 0 & 0 \\
0 & 0 & 0 & 0 \\
0 & 0 & 0 & 0 \\
0 & 0 & 0 & 0 \\
0 & 0 & 0 & 0 \\
0 & 0 & 0 & 0 \\
0 & 1.00 E^{+0} & 0 & 0 \\
0 & 0 & 1.00 E^{+0} & 0 \\
0 & 0 & 0 & 1.00 E^{+0} \\
1.00 E^{+0} & 0 & 0 & 0
\end{array}\right] \quad D_{G}=\left[\begin{array}{cccc}
0 & 0 & 0 & 0 \\
0 & 0 & 0 & 0 \\
0 & 0 & 0 & 0 \\
0 & 0 & 0 & 0
\end{array}\right]
\end{aligned}
$$




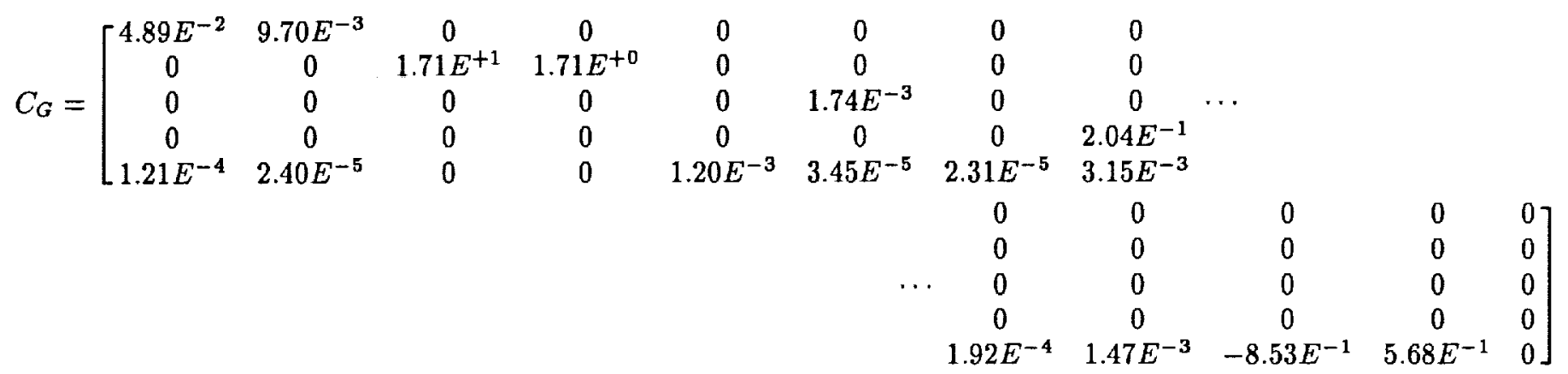

The centralized controller has state-space representation

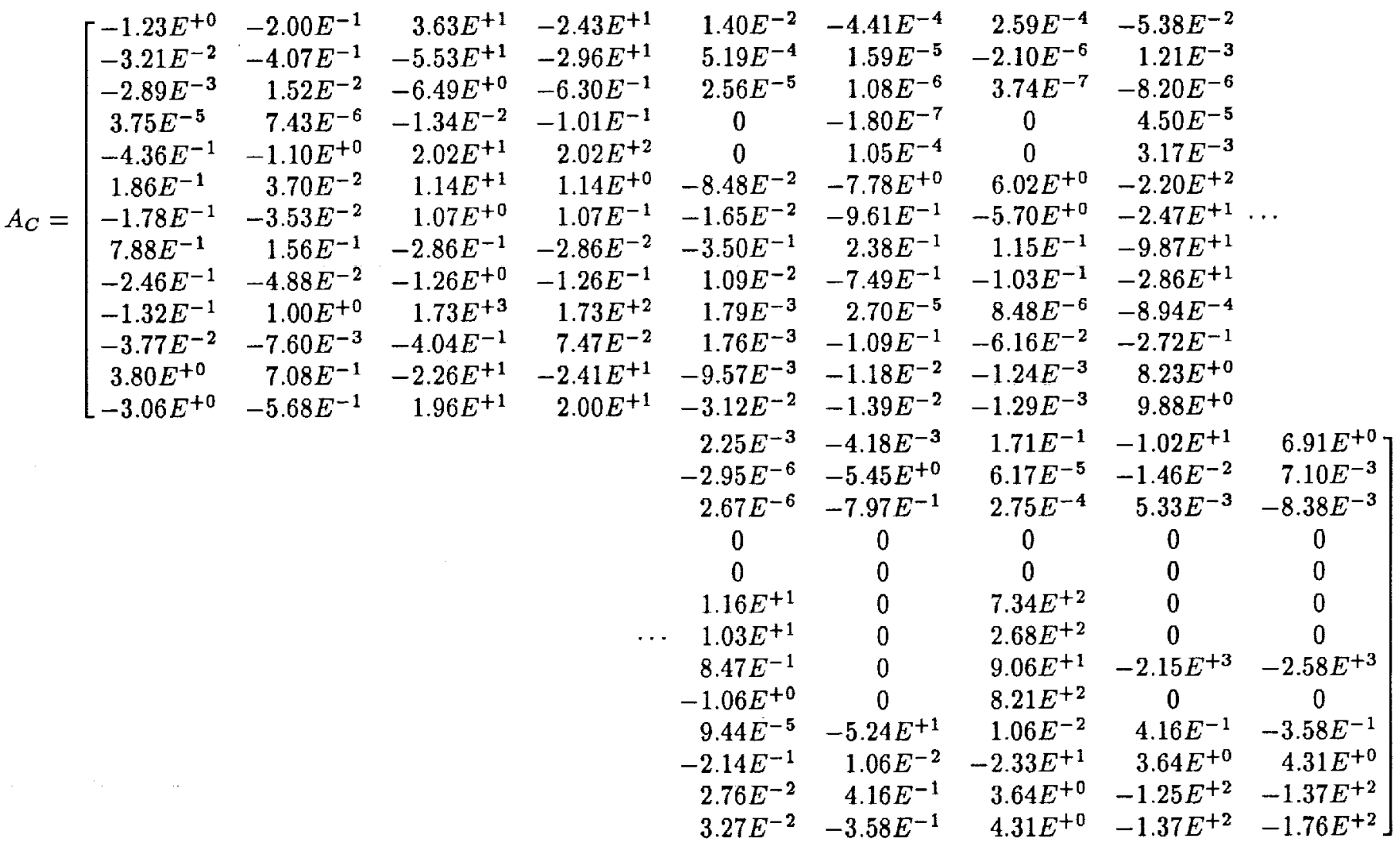

$$
\begin{aligned}
& B_{C}=\left[\begin{array}{rrrr}
2.43 E^{+1} & -4.36 E^{+0} & 4.33 E^{-1} & 4.51 E^{-1} \\
-3.99 E^{+0} & 1.45 E^{+1} & -1.81 E^{-2} & -5.07 E^{-3} \\
-4.13 E^{-3} & 3.66 E^{-1} & -7.81 E^{-5} & 2.19 E^{-4} \\
-7.66 E^{-4} & 5.89 E^{-2} & 1.03 E^{-4} & -2.20 E^{-4} \\
1.18 E^{+1} & -1.17 E^{+0} & -6.02 E^{-2} & -1.55 E^{-2} \\
1.20 E^{+1} & -6.68 E^{-1} & 2.05 E^{+3} & -6.00 E^{+2} \\
6.74 E^{+0} & -6.23 E^{-2} & 7.95 E^{+2} & 2.54 E^{+2} \\
1.08 E^{-1} & 1.66 E^{-2} & -5.12 E^{+0} & 4.16 E^{+1} \\
2.97 E^{+0} & 7.34 E^{-2} & 4.50 E^{+2} & 1.01 E^{+2} \\
-1.55 E^{-1} & -1.24 E^{+0} & 1.74 E^{-3} & 9.36 E^{-4} \\
1.05 E^{-2} & 6.63 E^{-4} & 1.16 E^{+0} & 4.47 E^{-1} \\
-9.50 E^{-1} & 1.18 E^{-1} & 2.94 E^{-1} & -7.46 E^{-1} \\
7.96 E^{-1} & -9.98 E^{-2} & 3.36 E^{-1} & -8.97 E^{-1}
\end{array}\right] \quad D_{C}=\left[\begin{array}{llll}
0 & 0 & 0 & 0 \\
0 & 0 & 0 & 0 \\
0 & 0 & 0 & 0 \\
0 & 0 & 0 & 0
\end{array}\right]
\end{aligned}
$$




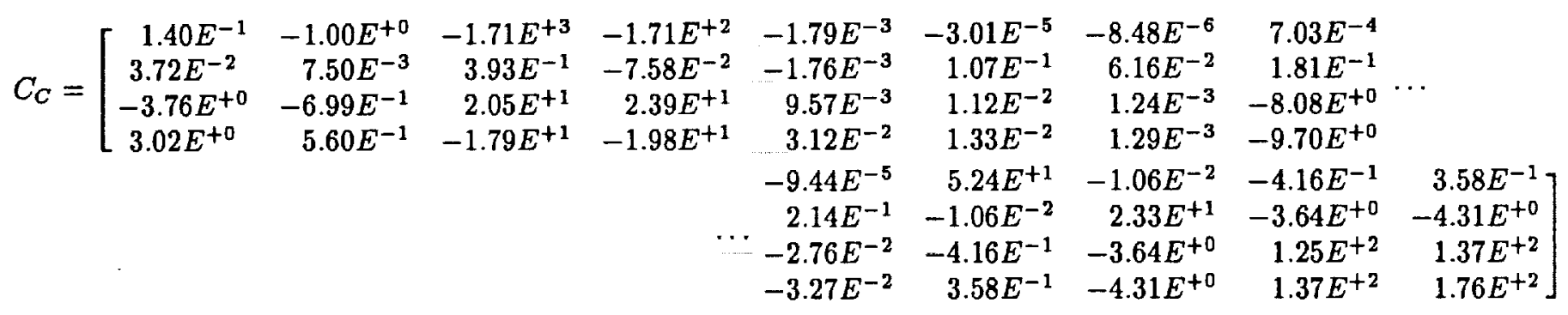

The initial partitioning consisted of and airframe controller with representation $S K_{A 0}$ of order 10 and an engine controller with representation $S K_{E 0}$ of order 7 . The initial partitioning was obtained by the procedure of reference [2]. This partitioning is not in the minimal parameter form. These are split because of their size.

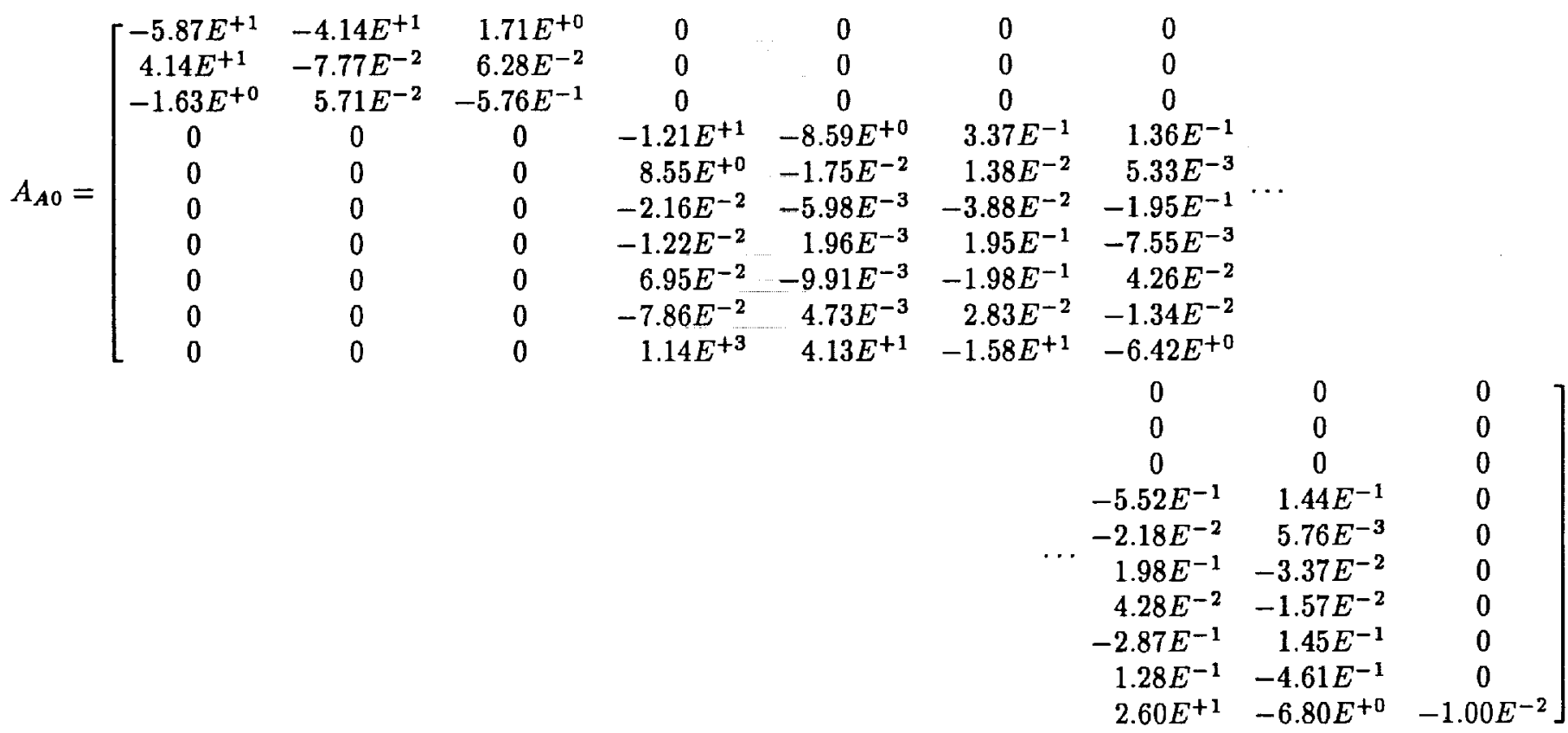

$$
\begin{aligned}
& B_{A 0}=\left[\begin{array}{rr}
2.90 E^{-1} & -2.68 E^{+1} \\
9.67 E^{-2} & 9.22 E^{-1} \\
1.19 E^{-1} & -3.63 E^{-1} \\
1.13 E^{+3} & -1.04 E^{+2} \\
-3.66 E^{+1} & 1.90 E^{+1} \\
1.51 E^{+0} & 1.58 E^{+1} \\
-3.36 E^{-1} & -6.41 E^{+0} \\
-3.35 E^{-1} & 2.59 E^{+1} \\
3.16 E^{+0} & -6.02 E^{+0} \\
0 & 0
\end{array}\right] \quad D_{A 0}=\left[\begin{array}{ll}
0 & 0 \\
0 & 0
\end{array}\right] \\
& C_{A 0}=\left[\begin{array}{cccccccccc}
2.68 E^{+1} & 9.27 E^{-1} & -3.83 E^{-1} & 0 & 0 & 0 & 0 & 0 & 0 & 0 \\
0 & 0 & 0 & 0 & 0 & 0 & 0 & 0 & 0 & 1.00 E^{-4}
\end{array}\right]
\end{aligned}
$$


The initial engine controller is represented by

$$
\begin{aligned}
& A_{E 0}=\left[\begin{array}{ccccccc}
-3.50 E^{+2} & 5.11 E^{+1} & 0 & 0 & 0 & 0 & 0 \\
-5.30 E^{+1} & -1.75 E^{-2} & 0 & 0 & 0 & 0 & 0 \\
0 & 0 & -1.67 E^{-1} & -1.90 E^{+1} & -1.04 E^{+0} & -4.51 E^{-1} & 2.83 E^{-1} \\
0 & 0 & 1.73 E^{+1} & -2.02 E^{+1} & -1.06 E^{+1} & -1.03 E^{+0} & -6.57 E^{-2} \\
0 & 0 & 1.17 E^{+0} & -1.06 E^{+1} & -1.92 E^{+1} & 1.89 E^{+1} & -2.99 E^{+1} \\
0 & 0 & -5.29 E^{+0} & 1.41 E^{+1} & 1.03 E^{+2} & -3.59 E^{+2} & 2.50 E^{+2} \\
0 & 0 & -8.39 E^{-1} & 5.67 E^{+0} & 3.56 E^{+1} & -2.46 E^{+2} & -2.07 E^{+1}
\end{array}\right] \\
& B_{E 0}=\left[\begin{array}{ccc}
-8.66 E^{+1} & 0 & 0 \\
-5.94 E^{-1} & 0 & 0 \\
0 & 1.69 E^{-2} & -1.83 E^{+0} \\
0 & -1.91 E^{+1} & 7.38 E^{-1} \\
0 & -5.75 E^{+0} & 5.45 E^{+0} \\
0 & 5.49 E^{+0} & -2.93 E^{+1} \\
0 & 2.51 E^{+0} & -4.48 E^{+0}
\end{array}\right] \quad D_{E 0}=\left[\begin{array}{lll}
0 & 0 & 0 \\
0 & 0 & 0 \\
0 & 0 & 0
\end{array}\right] \\
& C_{E 0}=\left[\begin{array}{crrrrrr}
-4.12 E^{-1} & -1.44 E^{-1} & -1.81 E^{+0} & -1.91 E^{+1} & -5.61 E^{+0} & -1.45 E^{+0} & 4.77 E^{-1} \\
7.47 E^{+1} & 1.45 E^{-1} & 1.86 E^{-1} & -6.92 E^{-1} & -3.67 E^{+0} & 1.91 E^{+1} & -3.44 E^{+0} \\
-4.37 E^{+1} & 5.58 E^{-1} & 2.15 E^{-1} & -9.85 E^{-1} & -4.22 E^{+0} & 2.28 E^{+1} & -3.79 E^{+0}
\end{array}\right]
\end{aligned}
$$

The parameter optimization algorithm for controller partitioning was applied to the problem with the starting matrices $S K_{A 0}$ and $S K_{E 0}$ listed above. The controllers obtained from this process had state-space representations $S K_{A o p t}$ for the airframe and $S K_{E o p t}$ for the engine.

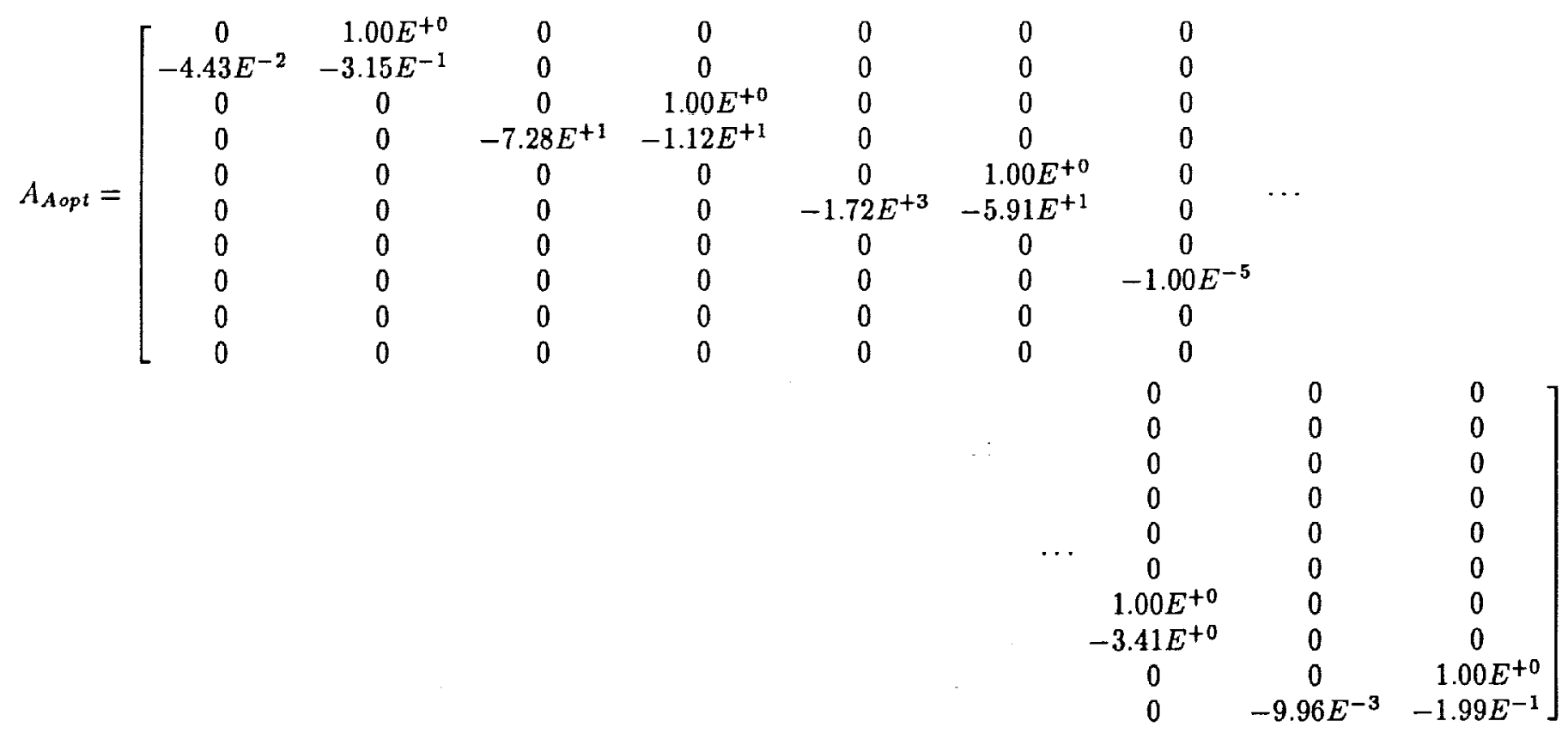




$$
\begin{aligned}
& B_{A o p t}=\left[\begin{array}{rr}
-3.67 E^{-3} & -5.45 E^{+3} \\
-1.24 E^{-2} & 1.60 E^{+3} \\
1.31 E^{+5} & -9.08 E^{+3} \\
8.50 E^{+4} & -2.58 E^{+4} \\
-4.23 E^{-1} & 2.03 E^{+1} \\
-6.84 E^{+0} & 9.54 E^{+2} \\
1.03 E^{+4} & -1.84 E^{+3} \\
-2.11 E^{+2} & 4.55 E^{+2}
\end{array}\right] \quad D_{A o p t}=\left[\begin{array}{ll}
2.82 E^{-1} & -2.94 E^{-3} \\
8.98 E^{-4} & -1.59 E^{-3}
\end{array}\right] \\
& C_{\text {Aopt }}=\left[\begin{array}{rrrrrrr}
-3.86 E^{-5} & -1.06 E^{-4} & -4.69 E^{-5} & -5.57 E^{-6} & -9.82 E^{+0} & -5.41 E^{-1} & -1.85 E^{-6} \\
2.49 E^{-4} & 2.03 E^{-3} & 1.91 E^{-6} & -1.28 E^{-5} & -2.99 E^{-3} & 2.17 E^{-4} & 7.65 E^{-5}
\end{array} \cdots\right. \\
& \left.\cdots \begin{array}{rrr}
4.09 E^{-4} & 6.44 E^{-2} & 6.46 E^{-1} \\
-2.63 E^{-4} & -1.59 E^{-2} & -1.57 E^{-1}
\end{array}\right]
\end{aligned}
$$

The optimized engine controller has state-space representation

$$
\begin{aligned}
& A_{\text {Eopt }}=\left[\begin{array}{ccccccc}
0 & 1.00 E^{+0} & 0 & 0 & 0 & 0 & 0 \\
-2.12 E^{+2} & -1.65 E^{+1} & 0 & 0 & 0 & 0 & 0 \\
0 & 0 & 0 & 1.00 E^{+0} & 0 & 0 & 0 \\
0 & 0 & -7.15 E^{+4} & -3.89 E^{+2} & 0 & 0 & 0 \\
0 & 0 & 0 & 0 & 0 & 1.00 E^{+0} & 0 \\
0 & 0 & 0 & 0 & -1.20 E^{+2} & -2.35 E^{+1} & 0 \\
0 & 0 & 0 & 0 & 0 & 0 & -3.41 E^{+2}
\end{array}\right] \\
& B_{E o p t}=\left[\begin{array}{rrr}
1.00 E^{-9} & -2.53 E^{+1} & 5.04 E^{+0} \\
1.00 E^{-9} & 9.38 E^{+1} & -7.78 E^{+1} \\
1.00 E^{-9} & 3.75 E^{+0} & -1.56 E^{+1} \\
1.00 E^{-9} & 1.67 E^{+3} & -7.69 E^{+3} \\
1.32 E^{+1} & -1.35 E^{+1} & 2.95 E^{+0} \\
-1.05 E^{+2} & 1.94 E^{+2} & -4.60 E^{+1}
\end{array}\right] \quad D_{E o p t}=\left[\begin{array}{rrr}
5.82 E^{-1} & 1.72 E^{+0} & 1.41 E^{-2} \\
-2.04 E^{+0} & 1.48 E^{+0} & 1.10 E^{-1} \\
6.64 E^{+0} & -6.93 E^{-1} & 1.03 E^{-2}
\end{array}\right] \\
& C_{E o p t}=\left[\begin{array}{crrrrrr}
-4.65 E^{+0} & -1.07 E^{+0} & -1.42 E^{-1} & -9.50 E^{-4} & 2.52 E^{+1} & 3.36 E^{+0} & 5.68 E^{-1} \\
1.97 E^{+0} & 1.44 E^{-1} & 8.80 E^{+0} & 5.88 E^{-2} & 2.14 E^{+1} & 1.46 E^{+0} & -7.44 E^{+1} \\
-1.62 E^{+0} & -1.10 E^{-1} & 1.01 E^{+1} & 6.27 E^{-2} & -1.92 E^{+1} & -1.37 E^{+0} & 4.47 E^{+1}
\end{array}\right]
\end{aligned}
$$

Residualization of high frequency modes was applied to the optimized engine controller to reduce it to one with order 4 . Balanced model reduction was applied to the optimized airframe controller to reduce it to one of order 6 . The optimization procedure was applied to this sixth order subcontroller with the engine controller fixed at the one of fourth order. The resulting reduced order optimized airframe subcontroller is

$$
S K_{\text {Ared }}=\left[\begin{array}{cccccc|rr}
0 & 1.00 E^{+0} & 0 & 0 & 0 & 0 & -2.81 E^{-1} & 8.98 E^{-2} \\
-2.84 E^{+0} & -4.39 E^{+1} & 0 & 0 & 0 & 0 & -1.39 E^{-2} & 1.18 E^{-1} \\
0 & 0 & 0 & 1.00 E^{+0} & 0 & 0 & 1.29 E^{+0} & -5.50 E^{+0} \\
0 & 0 & -1.71 E^{+3} & -6.19 E^{+1} & 0 & 0 & -1.53 E^{+1} & -9.18 E^{+2} \\
0 & 0 & 0 & 0 & 0 & 1.00 E^{+0} & 1.38 E^{+0} & -1.20 E^{-1} \\
0 & 0 & 0 & 0 & -6.92 E^{+1} & -1.18 E^{+1} & -5.03 E^{+0} & 5.95 E^{-1} \\
\hline-1.87 E^{-1} & -7.05 E^{+0} & 7.83 E^{+0} & 7.95 E^{-1} & 3.43 E^{+1} & 1.28 E^{+0} & -3.24 E^{+0} & 5.62 E^{-1} \\
-1.95 E^{+0} & 1.40 E^{+1} & 2.99 E^{-2} & 8.66 E^{-4} & -8.63 E^{+0} & -2.28 E^{+0} & 1.60 E^{-3} & -2.25 E^{-3}
\end{array}\right]
$$




\section{REFERENCES}

[1] Sanjay Garg, Peter J. Ouzts, Carl F. Lorenzo and Duane L. Mattern, IMPAC - An integrated methodology for propulsion and airframe control, Proceedings of the 1991 American Control Conference, June, 1991, Boston, MA, 1 (1991), 747- 754.

[2] Sanjay Garg, Controller Partitioning for Integrated Flight/Propulsion Control Implementation, presented at the 1992 American Control Conference, June, 1992, Chicago, IL.

[3] Phillip H. Schmidt and Sanjay Garg, Decentralized Hierarchical Partitioning of Centralized Integrated Controllers, Proceedings of the 1991 American Control Conference, Boston, MA, June, 1991 1 (1991), 755-760.

[4] Phillip H. Schmidt, Hierarchical Partitioning of Centralized Integrated Controllers, A Parameter Optimization Approach, in preparation, Technical Report of the Department of Mathematical Sciences, The University of Akron.

[5] B.A. Francis, A Course in $H^{\infty}$ Controls, Springer-Verlag, New York, Berlin, 1986.

[6] Uy-Loi Ly, Arthur E. Bryson and Robert H. Cannon, Design of low order compensators using parametric optimization, Automatica 21 (1985), 315-318.

[7] V. Mukhopadhyay and J. R. Newsom, A multiloop system stability margin study using matrix singular value, Journal of Guidance 7 (1987), 582-587.

[8] R. Fletcher, Practical Methods of Optimization, Wiley, New York, 1987.

[9] Sanjay Garg, Duane L. Mattern and Randy E. Bullard, Integrated fight/propulsion control system design based on a centralized approach, AIAA Paper No. 89-3519, Proceedings of the AIAA Guidance, Navigation and Control Conference, Aug. 1989, Boston, MA.

[10] B.C. Moore, Principal Component Analysis in Linear Systems: Controllability, Observability, and Model Reduction, IEEE Transactions on Automatic Control 26 (1981), 17-31.

[11] P.R. Apkarian, Structured Stability Robustness Improvement by Eigenspace Assignment Technigues: A Hybrid Methodology, Journal of Guidance, Control and Dynamics 12 (1981), 162-168.

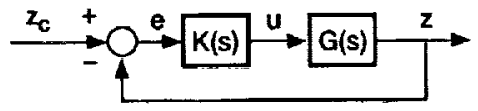

(a) Centralized control loop.

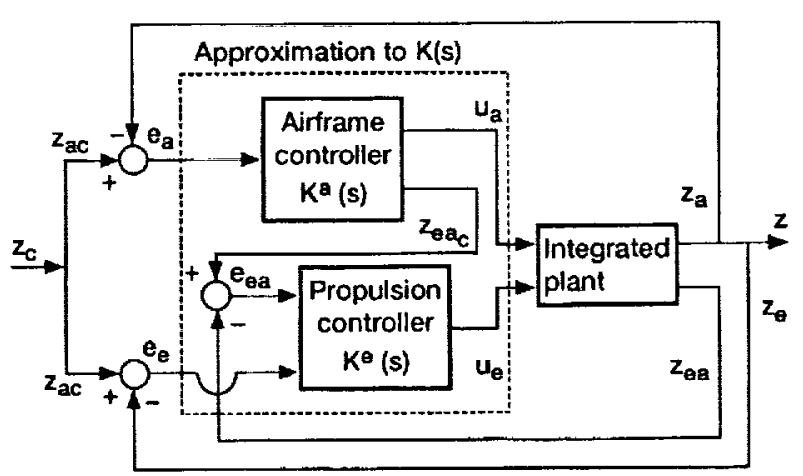

(b) Hierarchically decentralized control loop.

Figure 1.-Controller partitioning structure.

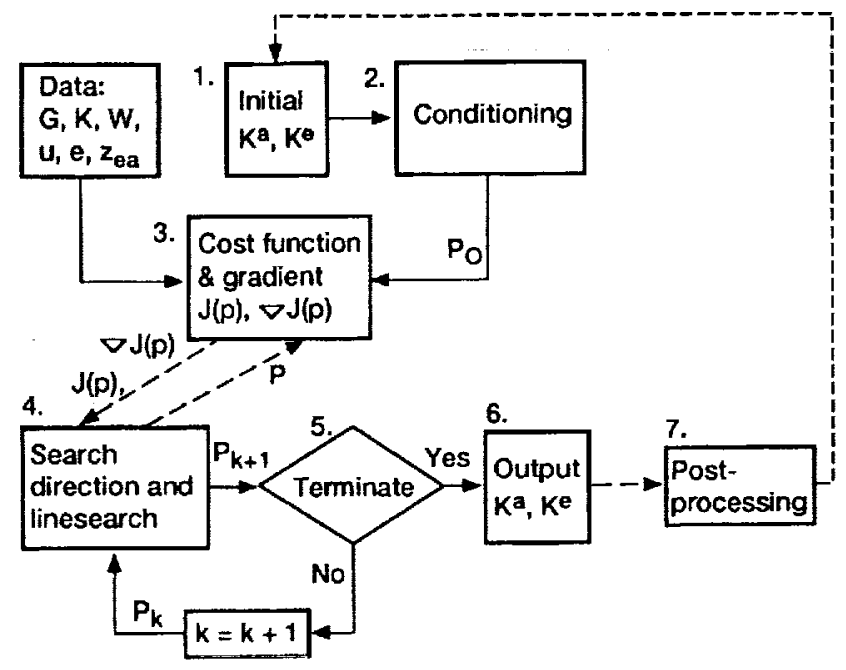

Figure 2.-Flowchart for partitioning optimization alagorithm. 


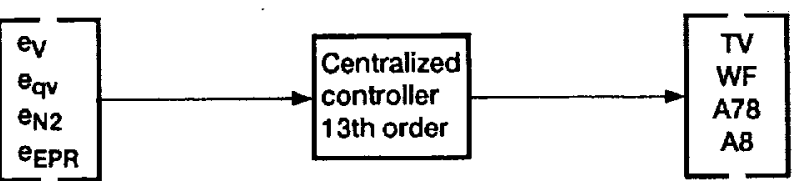

(a) Centralized control loop.

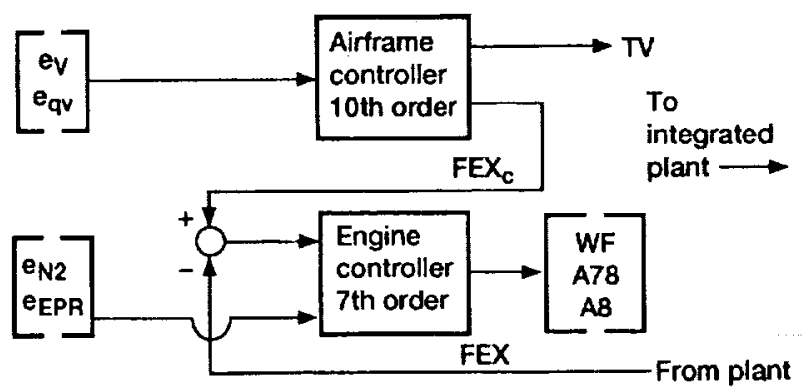

(b) Hierarchically decentralized control loop.

Figure 3.-Partitioned controller structure for design example.
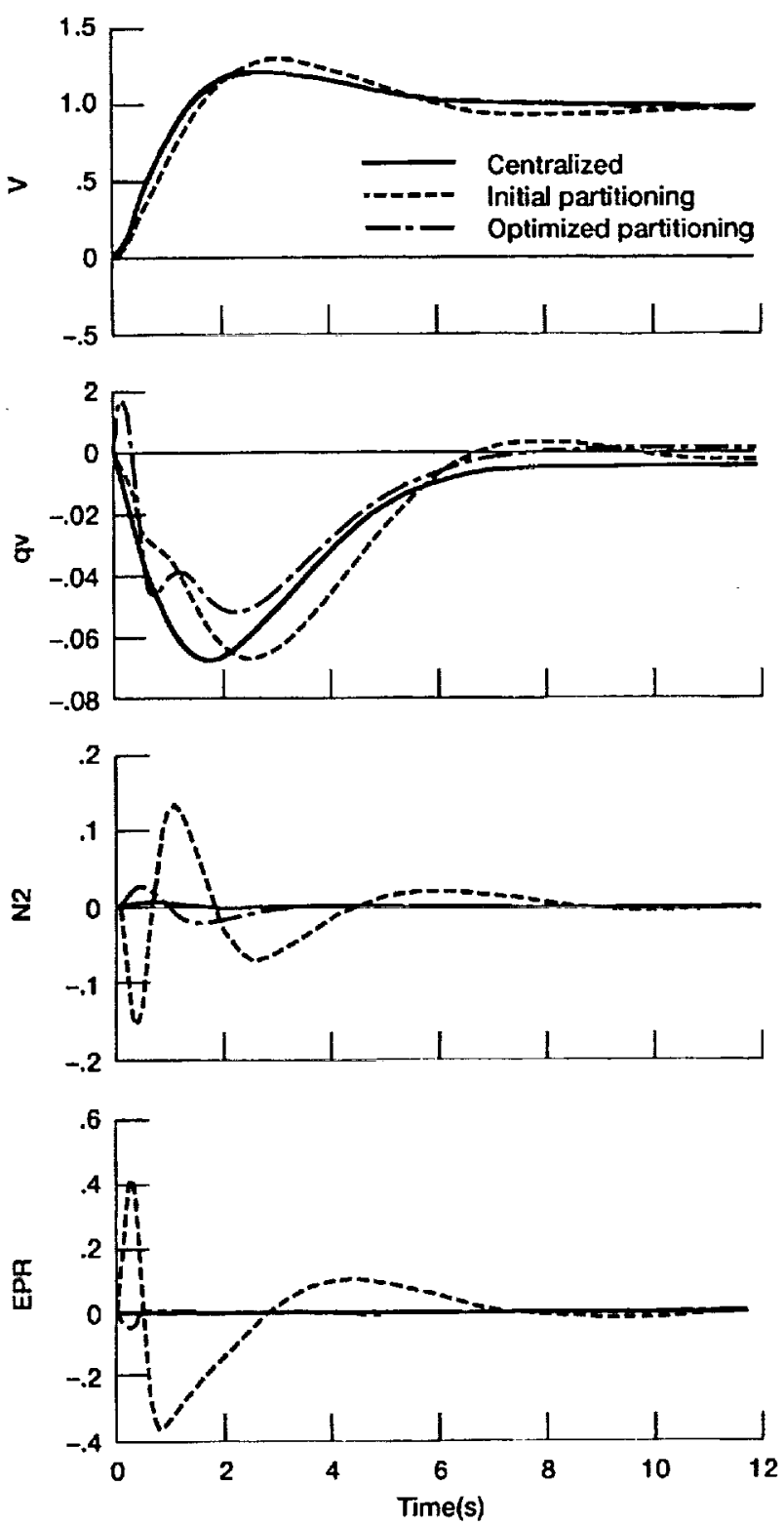

Figure 4.-Closed-loop system response to step velocity command for centralized controller, initial partitioning and optimized partitioning with $\lambda=0$. 


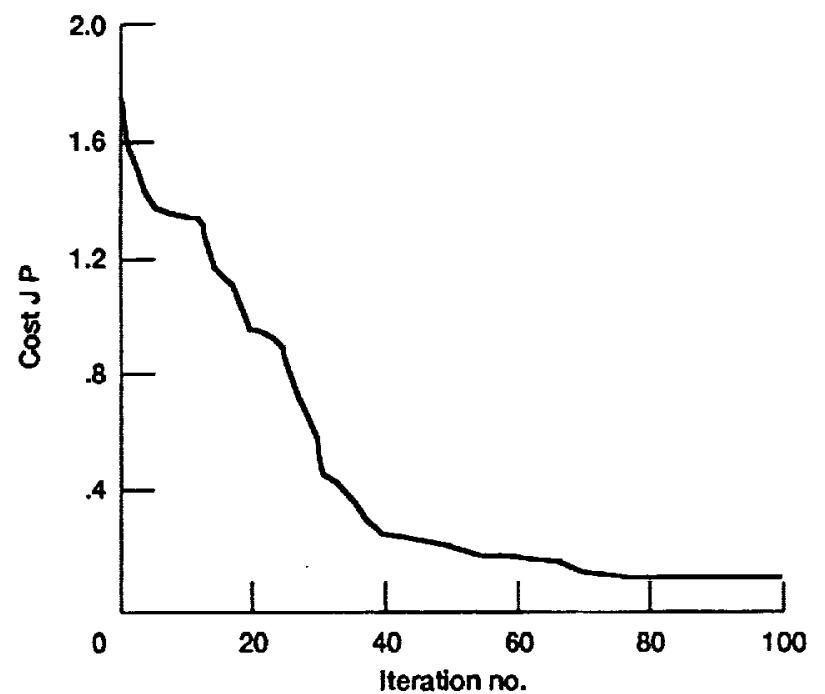

Figure 5.-Cost history for controller partitioning optimization with $\lambda=0$.

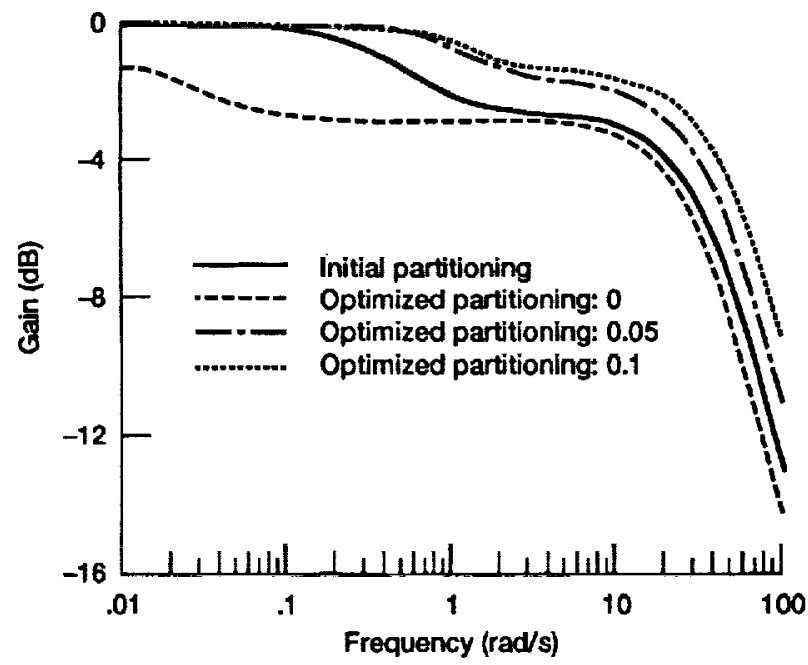

Figure 7.-Bode gain plot of engine closed-loop system response $\left(\mathrm{KEX}_{\mathrm{c}}-\mathrm{FEX}\right)$ for initial and optimlzed partitionings with varying $\lambda$.

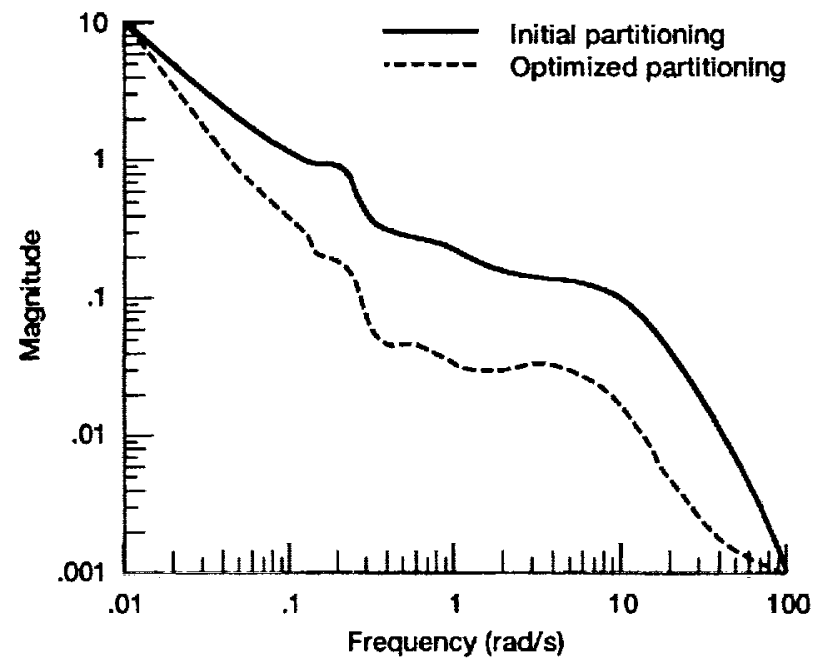

Figure 6.-Maximum singular value of weighted error, $\sigma_{\max }\left[(K-\bar{K}) G(I+K G)^{-1}\right](\omega)$, for initial and optimized $(\lambda=0)$ partitionings.

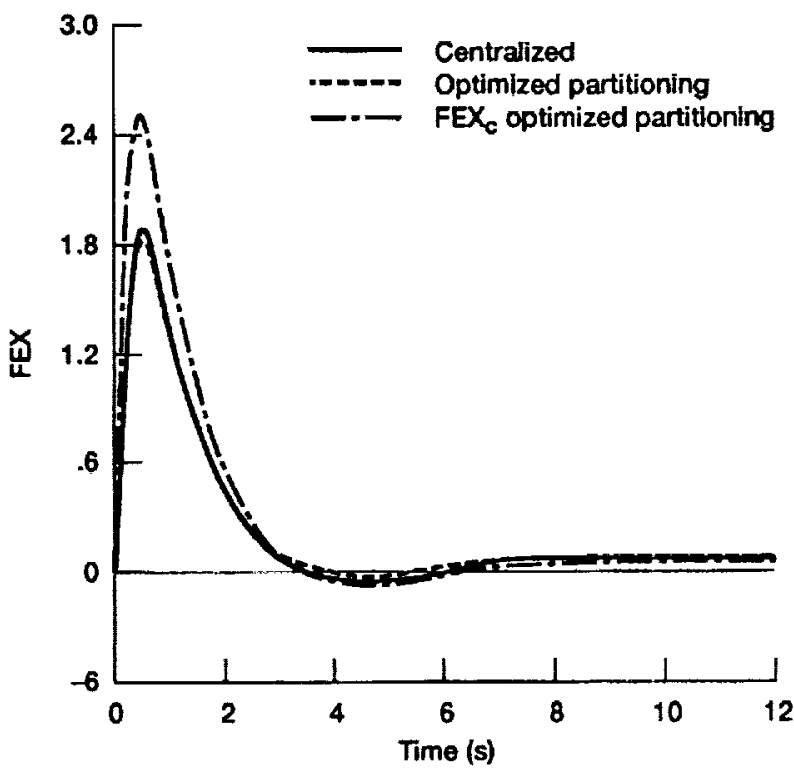

Figure 8.-FEX response to step velocity command for centralIzed and optimized partitioning with $\lambda=0$ and $F E X_{c}$ response for optimized controller $(\lambda=0)$. 


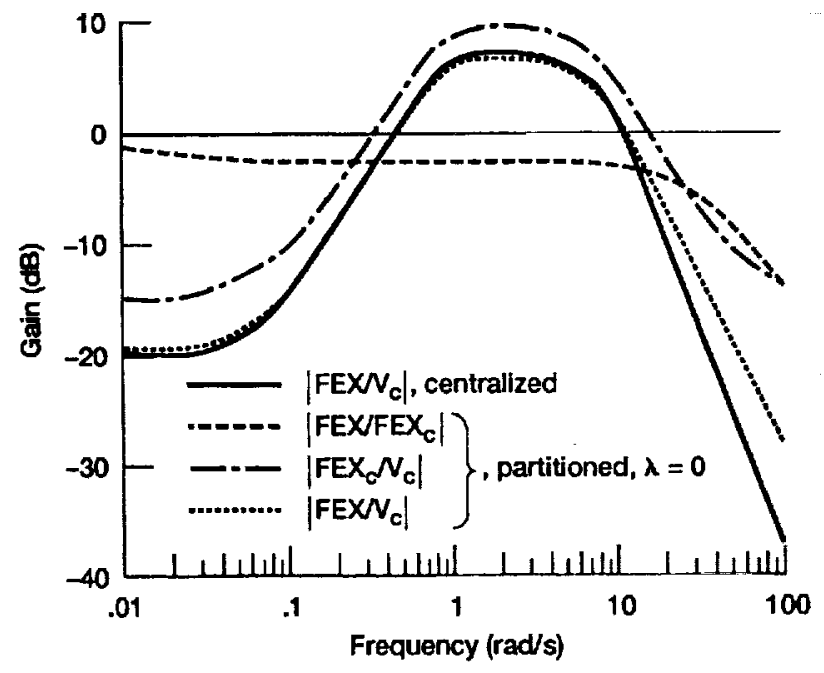

Figure 9.-Lead vs. bandwidth trade-off in controller partitioning.
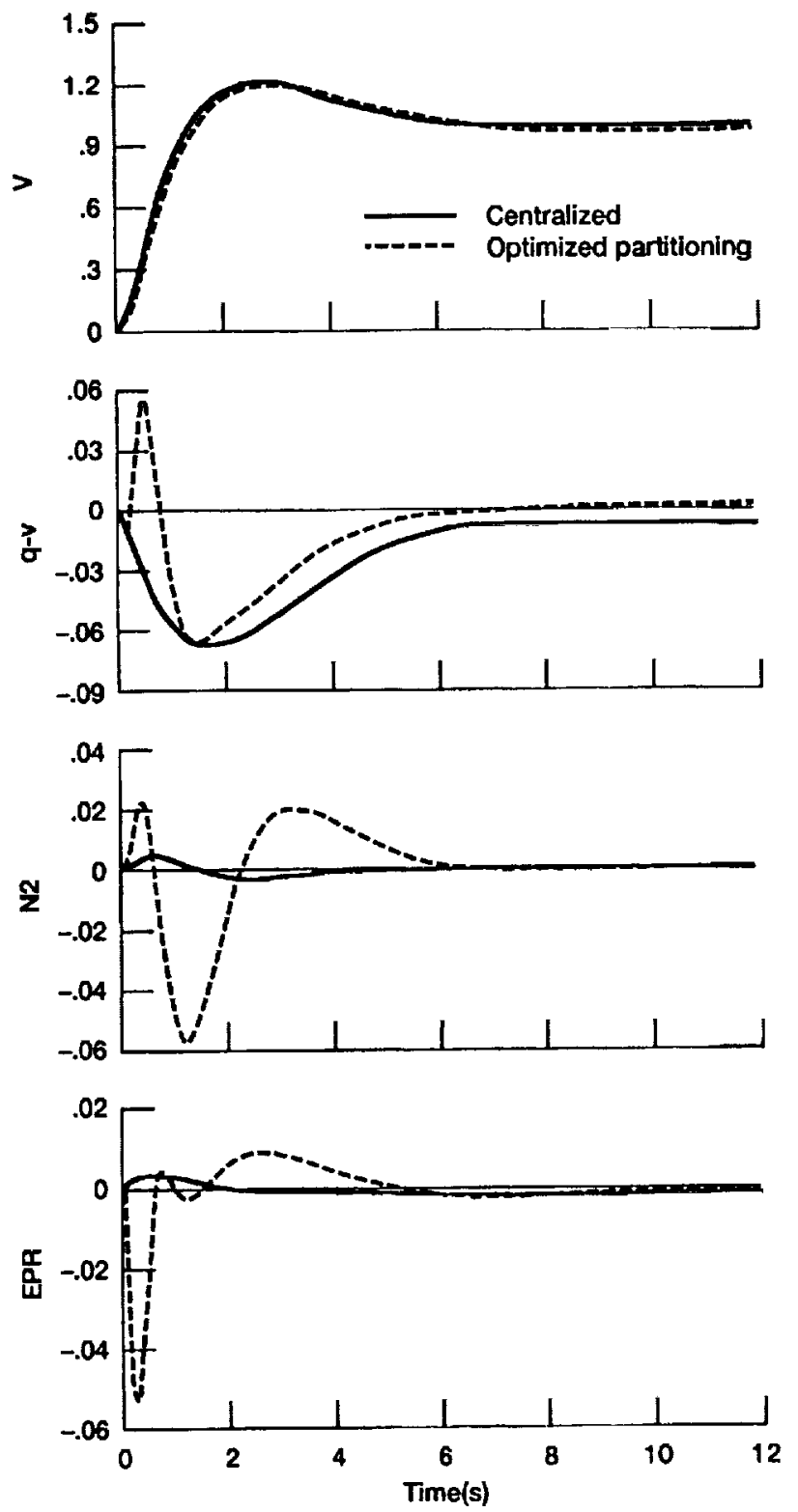

Figure 10.-Closed-loop system response to step velocity command for centralized controller and optimized partitioning with $\lambda=0.05$. 


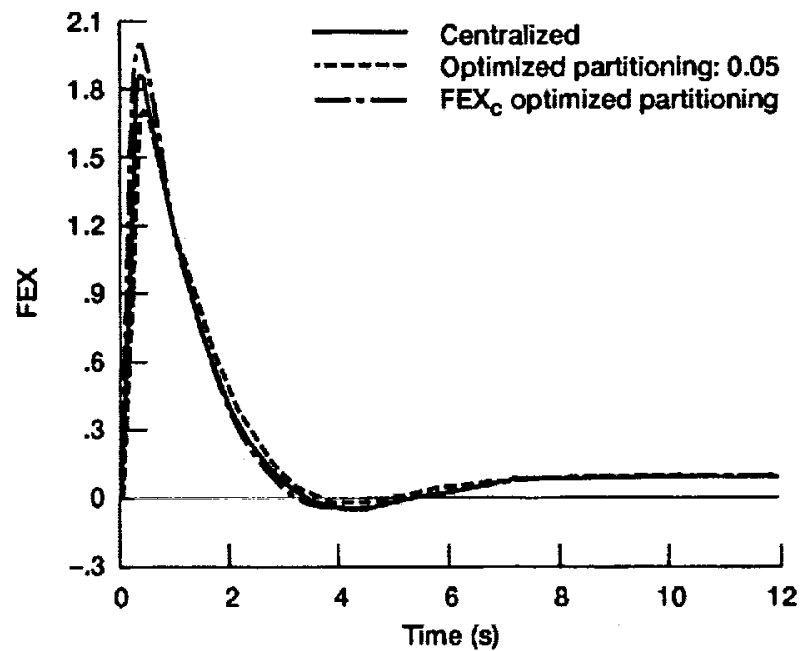

Figure 11.-FEX response to step velocity command for centralized controller and optimized partitioning with $\lambda=0.05$ and FEX $X_{c}$ response for optimized controller $(\lambda=0.05)$.
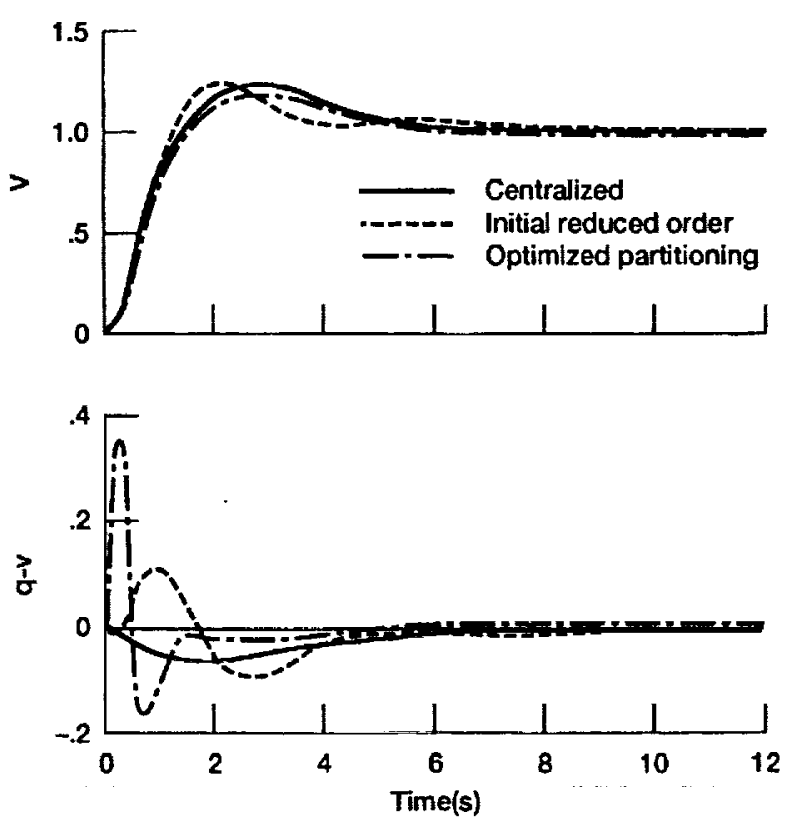

Figure 13.-Closed-loop system response to step velocity command for centralized controller and reduced order partition subcontrollers $\left(n_{a}=6 n_{c}=4\right)$ - initial and optimized ( $K^{\theta}$ fixed).

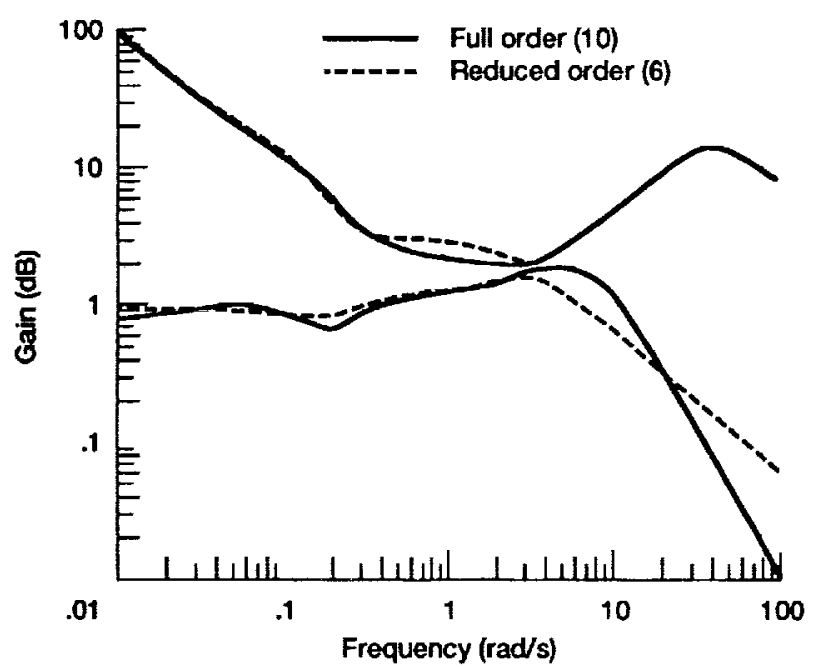

Figure 12. - Singular values of the airframe subcontroller for controller partitioning with $\lambda=0.05-$ full (10) and reduced (6) orders.

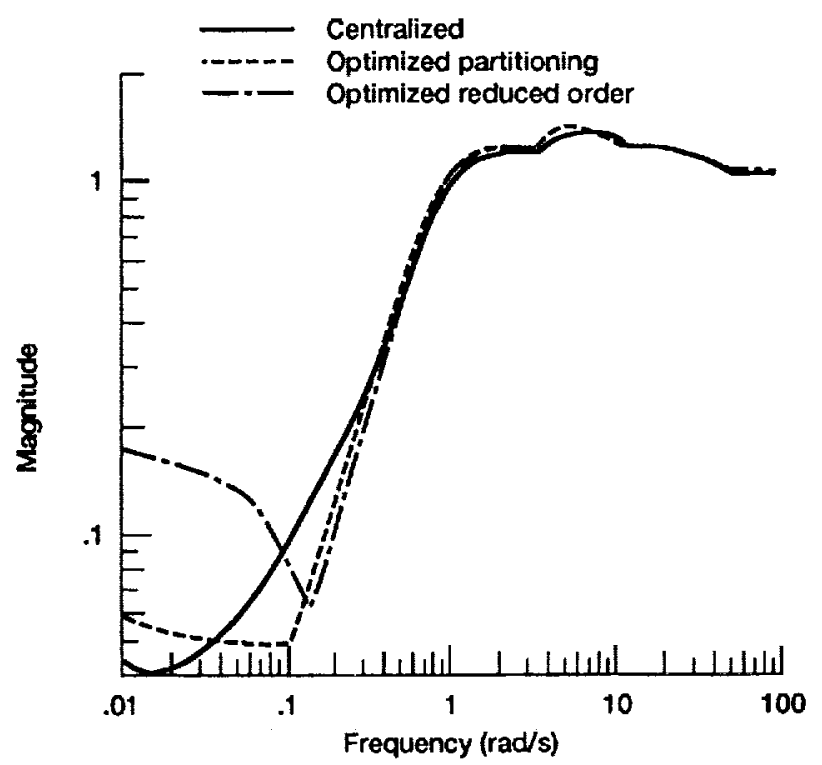

Figure 14. - Structured singular value for robustness analysis to gain and phase variations at the controlled outputs $\overline{\mathbf{z}}$ with centralized controller, for optimized partitioned controller $(\lambda=0.05)$, and optimized $\left(K^{\mathrm{B}}\right.$ fixed) reduced order controller. 

Public reporting burden for this collection of information is estimated to average 1 hour per response, including the time for reviewing instructions, searching existing data sources. gathering and maintaining the data needed, and completing and reviewing the collection of information. Send comments regarding this burden estimate or any other aspect of this collection of information, including suggestions for reducing this burden, to Washington Headquarters Services, Directorate for information Operations and Reports, 1215 Jefferson Davis Highway, Suite 1204, Arlington, VA 22202-4302, and to the Office of Management and Budget, Paperwork Reduction Project (0704-0188), Washington, DC 20503

\begin{tabular}{|l|c|c|}
\hline 1. AGENCY USE ONLY (Leave blank) & $\begin{array}{c}\text { 2. REPORT DATE } \\
\text { August } 1992\end{array}$ & $\begin{array}{r}\text { 3. REPOAT TYPE AND DATES COVERED } \\
\text { Technical Memorandum }\end{array}$
\end{tabular}

\section{TITLE AND SUBTITLE}

5. FUNDING NUMBERS

A Parameter Optimization Approach to Controller Partitioning for

Integrated Flight/Propulsion Control Application

6. AUTHOR(S)

WU-505-62-50

Phillip Schmidt, Sanjay Garg, and Brian Holowecky

7. PERFORMING ORgANIZATION NAME(S) AND ADDRESS(ES)

8. PERFORMING ORGANIZATION REPORT NUMBER

National Aeronautics and Space Administration

Lewis Research Center

Cleveland, Ohio 44135-3191

$E-7260$

9. SPONSORING/MONITORING AGENCY NAMES(S) AND ADDRESS(ES)

10. SPONSORING/MONITORING AGENCY REPORT NUMBER

National Aeronautics and Space Administration

Washington, D.C. 20546-0001

NASA TM-105826

11. SUPPLEMENTARY NOTES

Phillip Schmidt, The University of Akron, Akron, Ohio 44325; Sanjay Garg, Lewis Research Center, Cleveland, Ohio; and Brian Holowecky, The University of Akron, Akron, Ohio 44325. Responsible person, Sanjay Garg, (216) 433-2355.

12a. DISTRIBUTION/AVAILABILITY STATEMENT 12b. DISTAIBUTION CODE

Unclassified - Unlimited

Subject Categories 63 and 08

13. ABSTRACT (Maximum 200 words)

A parameter optimization framework is presented to solve the problem of partitioning a centralized controller into a decentralized hierarchical structure suitable for integrated flight/propulsion control implementation. The controller partitioning problem is briefly discussed and a cost function to be minimized is formulated, such that the resulting "optimal" partitioned subsystem controllers will closely match the performance (including robustness) properties of the closed-loop system with the centralized controller while maintaining the desired controller partitioning structure. The cost function is written in terms of parameters in a state-space representation of the partitioned subcontrollers. Analytical expressions are obtained for the gradient of this cost function with respect to parameters and an optimization algorithm is developed using modern computer-aided control design and analysis software. The capabilities of the algorithm are demonstrated by application to partitioned integrated flight/propulsion control design for a modern fighter aircraft in the short approach to landing task. The partitioning optimization is shown to lead to reduced-order subcontrollers that match the closed-loop command tracking and decoupling performance achieved by a high-order centralized controller.

14. SUBJECT TERMS

Controller partitioning; Integrated control; Centralized control; Decentralized control; Parameter optimization

\begin{tabular}{|c|c|}
\hline 17. SECUAITY CLASSIFICATION & 18. SECURITY CLASSIFICATION \\
OF REPORT & OF THIS PAGE \\
Unclassified & Unclassified
\end{tabular}

NSN 7540-01-280-5500
19. SECURITY CLASSIFICATION OF ABSTRACT

Unclassified
15. NUMBER OF PAGES

30

16. PRICE CODE $\mathrm{AO} 3$

20. LIMITATION OF ABSTRACT Standard Form 298 (Rev. 2-89) Prescribed by ANSI SId. Z39-18 298-102 